\title{
CUSPS OF THE KÄHLER MODULI SPACE AND STABILITY CONDITIONS ON K3 SURFACES
}

\author{
HEINRICH HARTMANN
}

\begin{abstract}
In Ma09] S. Ma established a bijection between FourierMukai partners of a K3 surface and cusps of the Kähler moduli space. The Kähler moduli space can be described as a quotient of Bridgeland's stability manifold. We study the relation between stability conditions $\sigma$ near to a cusp and the associated Fourier-Mukai partner $Y$ in the following ways. (1) We compare the heart of $\sigma$ to the heart of coherent sheaves on $Y$. (2) We construct $Y$ as moduli space of $\sigma$-stable objects. An appendix is devoted to the group of auto-equivalences of $\mathcal{D}^{b}(X)$ which respect the component $\operatorname{Stab}^{\dagger}(X)$ of the stability manifold.
\end{abstract}

\section{Contents}

1. Introduction

2. Geometry of the Mukai lattice

3. Ma's Theorem

4. Stability conditions

5. Cusps and hearts of stability conditions

6. Moduli spaces of complexes on K3 surfaces

7. Appendix: Equivalences respecting $\operatorname{Stab}^{\dagger}(X)$

References

\section{INTRODUCTION}

Let $X$ be a projective K3 surface over the complex numbers, and let $\mathcal{T}=\mathcal{D}^{b}(X)$ be the bounded derived category of coherent sheaves on $X$.

We associate to $\mathcal{T}$ the complexified Kähler moduli space $K M(\mathcal{T})$ by the following procedure. Let $N(\mathcal{T})$ be the numerical Grothendieck group of $\mathcal{T}$ endowed with the (negative) Euler pairing. We consider the following period domain

$$
\mathfrak{D}(\mathcal{T})=\left\{[z] \in \mathbb{P}\left(N(\mathcal{T})_{\mathbb{C}}\right) \mid z . z=0, z . \bar{z}>0\right\} .
$$

and define $K M(\mathcal{T})$ to be a connected component of $\operatorname{Aut}(\mathcal{T}) \backslash \mathfrak{D}(\mathcal{T})$. The image of $\operatorname{Aut}(\mathcal{T})$ in the orthogonal group $O(N(\mathcal{T}))$ is known by HMS09. In particular it is an arithmetic subgroup. Therefore, we can compactify the Kähler moduli space to a projective variety $\overline{K M}(\mathcal{T})$ using the Baily-Borel construction [BB66]. 
The boundary $\overline{K M}(\mathcal{T}) \backslash K M(\mathcal{T})$ consist of components, called cusps, which are divided into the following types (cf. section 3 ):

- 0-dimensional standard cusps,

- 0-dimensional cusps of higher divisibility and

- 1-dimensional boundary components.

In [Ma09, Ma10] Shouhei Ma establishes a bijection between

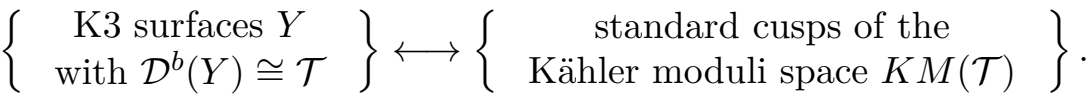

Moreover, cusps of higher divisibility correspond to realizations of $\mathcal{T}$ as the derived category of sheaves on a K3 surface twisted by a Brauer class. Unfortunately the proof is not geometric but uses deep theorems due to Mukai and Orlov to translate the statement into lattice theory.

The aim of this work is to find a more geometric explanation for this phenomenon using Bridgeland stability conditions [Bri07, Bri08. The space $\operatorname{Stab}(\mathcal{T})$ of Bridgeland stability conditions on $\mathcal{T}$ is a complex manifold and carries canonical actions of $A u t(\mathcal{T})$ and of the universal cover $\widetilde{G l}_{2}^{+}(\mathbb{R})$ of $G l_{2}^{+}(\mathbb{R})$. For each pair of $\omega \in A m p(X)$ and $\beta \in N S(X)_{\mathbb{R}}$ with $\omega^{2}>2$ Bridgeland constructs an explicit stability conditions $\sigma_{X}(\beta, \omega) \in \operatorname{Stab}(\mathcal{T})$. Denote by $\operatorname{Stab} b^{\dagger}(\mathcal{T})$ the connected component of $\operatorname{Stab}(\mathcal{T})$ containing these stability conditions.

A special open subset of $K M(\mathcal{T})$ can be identified with the quotient space

$$
K M_{0}(\mathcal{T}) \cong A u t^{\dagger}(\mathcal{T}) \backslash S t a b^{\dagger}(\mathcal{T}) / \widetilde{G l}_{2}^{+}(\mathbb{R}),
$$

where $A u t^{\dagger}(\mathcal{T})$ is the group of auto-equivalences respecting the distinguished component $\operatorname{Stab}^{\dagger}(\mathcal{T})$. This statement is essentially due to Bridgeland and was stated in [Ma09] and [Bri09] before. However, it seems to rely on properties of the group $A u t^{\dagger}(\mathcal{T})$ which are established in appendix [7, cf. Corollary 7.10. We denote the quotient map by $\pi: \operatorname{Stab}^{\dagger}(\mathcal{T}) \rightarrow K M(\mathcal{T})$.

1.1. Hearts of stability conditions. Our first result addresses the following question: Every stability condition $\sigma$ determines a heart $\mathcal{A}(\sigma)$ of a bounded t-structure. Also, every derived equivalence $\Phi: \mathcal{D}^{b}(Y) \stackrel{\sim}{\longrightarrow} \mathcal{T}$ determines the heart $\Phi(\operatorname{Coh}(Y))$. How are these two hearts related for $\sigma$ near the cusp associated to $Y$ ?

Theorem (5.5)). Let $[v] \in \overline{K M}(\mathcal{T})$ be a standard cusp, and $Y$ the $K 3$ surface associated to $[v]$ by $\left(^{*}\right)$. Then, there exists a path $\sigma(t) \in \operatorname{Stab}^{\dagger}(\mathcal{T}), t \gg 0$ and an equivalence $\Phi: \mathcal{D}^{b}(Y) \stackrel{\sim}{\longrightarrow} \mathcal{T}$ such that

(1) $\lim _{t \rightarrow \infty} \pi(\sigma(t))=[v] \in \overline{K M}(\mathcal{T})$ and

(2) $\lim _{t \rightarrow \infty} \mathcal{A}(\sigma(t))=\Phi(\operatorname{Coh}(Y))$ as subcategories of $\mathcal{T}$.

The path in this theorem is the image of $\sigma_{Y}(t \beta, t \omega)$ under a certain equivalence. It is easy to construct other paths satisfying (1) which have limiting hearts given by tilts of $\operatorname{Coh}(Y)$. The natural question arises, how all limiting hearts look like.

Instead of allowing all possible paths we identify a class of paths $\gamma(t) \epsilon$ $K M(\mathcal{T})$, called linear degenerations to a cusp $[v] \in \overline{K M}(\mathcal{T})$, and restrict our attention to them. The prototypical example of a linear degeneration is 


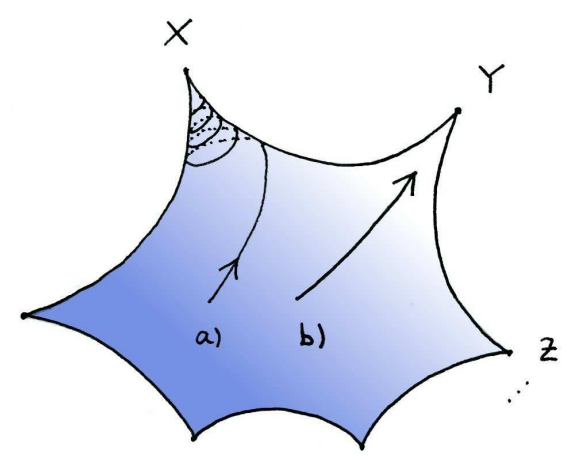

FIgURE 1. Kähler moduli space with cusps, associated K3 surfaces and two different degenerating paths. Paths of type a) are considered in Theorem 5.5, whereas b) pictures a linear degeneration.

$\pi\left(\sigma_{X}(\beta, t \omega)\right)$. In this case the heart of $\sigma_{X}(\beta, t \omega)$ is constant and given by an explicit tilt of $\operatorname{Coh}(X)$. We prove the following proposition.

Proposition (5.9, 5.2). Let $[v] \in \overline{K M}(\mathcal{T})$ be a standard cusp and $\gamma(t) \in$ $K M(\mathcal{T})$ be a linear degeneration to $[v]$, then $\gamma(t)$ is a geodesic converging to $[v]$.

The (orbifold-)Riemannian metric we use is induced via the isomorphism $\mathfrak{D}(\mathcal{T}) \cong O(2, \rho) / S O(2) \times O(\rho)$.

Conjecture. Every geodesic converging to $[v]$ is a linear degeneration.

This conjecture is true in the case that $X$ has Picard rank one. Moreover, if one uses the Borel-Serre compactification to compactify $K M(\mathcal{T})$ the conjecture seems to follow from [JM02].

The next theorem classifies paths of stability conditions mapping to linear degenerations in the Kähler moduli space.

Theorem (5.3). Let $[v]$ be a standard cusp of $\overline{K M}(\mathcal{T})$. Let $\sigma(t) \in \operatorname{Stab}^{\dagger}(X)$ be a path in the stability manifold such that $\pi(\sigma(t)) \in \overline{K M}(X)$ is a linear degeneration to $[v]$. Let $Y$ be the K3 surface associated to $[v]$ by $\left(^{*}\right)$. Then there exist

(1) a derived equivalence $\Phi: \mathcal{D}^{b}(Y) \stackrel{\sim}{\longrightarrow} \mathcal{T}$

(2) classes $\beta \in N S(Y)_{\mathbb{R}}, \omega \in \overline{A m p}(Y)$ and

(3) a path $g(t) \in \widetilde{G l} l_{2}^{+}(\mathbb{R})$

such that

$$
\sigma(t)=\Phi_{*}\left(\sigma_{Y}^{*}(\beta, t \omega) \cdot g(t)\right)
$$

for all $t \gg 0$.

Moreover, the hearts of $\sigma(t) \cdot g(t)^{-1}$ are independent of $t$ for $t \gg 0$. If $\omega \in \operatorname{Amp}(X)$, then the heart can be explicitly described as a tilt of $\operatorname{Coh}(Y)$.

Here, $\sigma_{X}^{*}(\beta, \omega)$ is an extension of Bridgeland's construction of $\sigma_{X}(\beta, \omega)$ to the case that $\omega \in \overline{A m p}(X)$ and $\omega^{2}>2$ (cf. Lemma 4.8). 
1.2. Moduli spaces. Another question we posed ourselves is: Can we construct $Y$ as a moduli space of stable objects in stability conditions near the associated cusp $[v] \in \overline{K M}(\mathcal{T})$ ?

For $v \in N(X)$ and $\sigma \in \operatorname{Stab}(X)$ we consider the following moduli space of semi-stable objects

$$
\mathcal{M}^{\sigma}(v)=\left\{E \in \mathcal{D}^{b}(X) \mid E \sigma \text {-semi-stable, } v(E)=v\right\} / \sim
$$

where $E \sim F$ if there is an even number $k \in 2 \mathbb{Z}$ and a quasi-isomorphism $E \cong F[k]$. This is a version of the moduli stack constructed by Lieblich [Lie06] and Toda [Tod08]. We prove the following result.

Theorem (6.12). If $v \in N(X)$ is an isotropic vector with $v \cdot N(X)=\mathbb{Z}$ and $\sigma \in \operatorname{Stab}^{\dagger}(X)$ a v-general stability condition, then:

(1) The moduli space $\mathcal{M}^{\sigma}(v)$ is represented by a K3 surface $Y$.

(2) The Hodge structure $H^{2}(Y, \mathbb{Z})$ is the isomorphic to the subquotient of $\widetilde{H}(X, \mathbb{Z})$ given by $v^{\perp} / \mathbb{Z} v$.

(3) The universal family $E \in \mathcal{M}_{X}^{\sigma}(v)(Y) \subset \mathcal{D}^{b}(X \times Y)$ induces a derived equivalence $\mathcal{D}^{b}(X) \stackrel{\sim}{\longrightarrow} \mathcal{D}^{b}(Y)$.

This is in some sense a negative answer to our question: The isomorphism type of $\mathcal{M}^{\sigma}(v)$ does not depend on whether the stability condition $\sigma$ is close to a cusp or not. On the other hand, the isotropic vector $v$ determines a standard cusp $[v] \in \overline{K M}(\mathcal{T})$ and $Y$ is indeed the K3 surface associated to the cups.

1.3. Auto-equivalences. On the way of proving the above result we need to construct enough equivalences that respect the distinguished component $S t a b^{\dagger}(\mathcal{T})$. We collected our results in an appendix which is essentially independent of the rest of the paper.

Theorem (7.5, 7.6, 77.7). The following equivalences respect the distinguished component.

- For a fine, compact, two-dimensional moduli space of Gieseker-stable sheaves $M^{h}(v)$, the Fourier-Mukai equivalence induced by the universal family.

- The spherical twists along Gieseker-stable spherical vector bundles.

- The spherical twists along $\mathcal{O}_{C}(k)$ for a $(-2)$-curve $C \subset X$ and $k \in \mathbb{Z}$.

This allows us to show the following strengthening of a result of [HLOY04, HMS09.

Proposition (7.9). Let $A u t^{\dagger}\left(\mathcal{D}^{b}(X)\right) \subset \operatorname{Aut}\left(\mathcal{D}^{b}(X)\right)$ be the subgroup of auto-equivalences which respect the distinguished component. Then

$$
A u t^{\dagger}\left(\mathcal{D}^{b}(X)\right) \longrightarrow O_{\text {Hodge }}^{+}(\tilde{H}(X, \mathbb{Z}))
$$

is surjective.

Another direct consequence is the description (1) of the Kähler moduli space, cf. Corollary 7.10. 
Acknowledgment. This work is part of a Ph.D. thesis written under the supervision of Prof. D. Huybrechts in Bonn whom we thank sincerely for his generous support.

We thank T. Bridgeland, E. Looijenga, N. Perrin, M. Rapoport, D. van Straten for their help with various questions and P. Sosna for his careful reading of earlier versions of this text. We thank the Bonn International Graduate School in Mathematics and the Sonderforschungsbereich TR 45 for financial support.

1.4. Notation. Our notation will largely follow Huybrechts' book Huy06]. Let $X$ be a projective K3 surface over the complex numbers. The Picard rank of $X$ is denoted by $\rho(X)=r k(N S(X))$.

We write $\tilde{H}(X, \mathbb{Z})$ for the full cohomology $H^{0}(X, \mathbb{Z}) \oplus H^{2}(X, \mathbb{Z}) \oplus H^{4}(X, \mathbb{Z})$ endowed with the Mukai pairing $(r, l, s) \cdot\left(r^{\prime}, l^{\prime}, s^{\prime}\right)=l . l^{\prime}-r s^{\prime}-r^{\prime} s$, and the weight-two Hodge structure

$$
\begin{aligned}
\tilde{H}^{1,1}(X) & =H^{0,0}(X) \oplus H^{1,1}(X) \oplus H^{2,2}(X), \\
\tilde{H}^{2,0}(X) & =H^{2,0}(X), \quad \tilde{H}^{0,2}(X)=H^{0,2}(X) .
\end{aligned}
$$

We write $N(X)=H^{0}(X, \mathbb{Z}) \oplus N S(X) \oplus H^{4}(X, \mathbb{Z})$ for the extended NéronSeveri group. It is an even lattice of signature $(2, \rho(X))$.

To a sheaf $A \in \operatorname{Coh}(X)$ we associate the Mukai vector

$$
v(A)=\sqrt{\operatorname{td}(X)} \cdot \operatorname{ch}(A)=\left(r(A), c_{1}(A), s(A)\right) \in N(X),
$$

where $s(A)=\frac{1}{2} c_{1}(A)^{2}-c_{2}(A)+r(A)$. By the Riemann-Roch theorem we have $-\chi(A, B)=v(A) \cdot v(B)$. Therefore, we can identify $N(X)$ with the numerical Grothendieck group $N(\operatorname{Coh}(X))=K(\operatorname{Coh}(X)) / \operatorname{rad}(\chi)$ via the map $A \mapsto v(A)$.

We denote by $\mathcal{D}^{b}(X)$ the bounded derived category of coherent sheaves on $X$. We have natural isomorphisms between the numerical Grothendieck groups $N\left(\mathcal{D}^{b}(X)\right) \cong N(\operatorname{Coh}(X)) \cong N(X)$. Every $\mathbb{C}$-linear, exact equivalence $\Phi: \mathcal{D}^{b}(X) \rightarrow \mathcal{D}^{b}(Y)$ induces a Hodge isometry which we denote by

$$
\Phi^{H}: \tilde{H}(X, \mathbb{Z}) \longrightarrow \tilde{H}(Y, \mathbb{Z}) .
$$

We say that two K3 surfaces $X$ and $Y$ are derived equivalent if $\mathcal{D}^{b}(X)$ is equivalent to $\mathcal{D}^{b}(Y)$ as a $\mathbb{C}$-linear, triangulated category.

\section{Geometry of the Mukai lattice}

Let $X$ be a projective $\mathrm{K} 3$ surface over the complex numbers and $\mathcal{D}^{b}(X)$ its derived category. Let $N=N\left(\mathcal{D}^{b}(X)\right)$ be the numerical Grothendieck group of $\mathcal{D}^{b}(X)$. In this section, we will introduce various groups and spaces that are naturally associated to the lattice $N$.

The isomorphism $N \cong N(X)$ gives us the following extra structures.

(1) An isotropic vector $v_{0}=(0,0,1) \in N$.

(2) An embedding of a hyperbolic plane

$$
\varphi: U \cong H^{0}(X, \mathbb{Z}) \oplus H^{4}(X, \mathbb{Z}) \rightarrow N .
$$

(3) The choice of an ample chamber $A m p(X) \subset N S(X)_{\mathbb{R}} \subset N_{\mathbb{R}}$.

(4) A weight-two Hodge structure $\tilde{H}(X, \mathbb{Z})$ with $\tilde{H}^{1,1}(X) \cap \tilde{H}(X, \mathbb{Z})=$ $N$. In particular, a group action of $O_{\text {Hodge }}(\tilde{H}(X, \mathbb{Z}))$ on $N$. 
We will pay special attention to which constructions depend on what additional data.

Convention 2.1. In later sections, when we have fixed an identification $N=$ $N(X)$, we will allow ourselves to abuse the notation by filling in the standard choices of the above extra structures. For example, we shall write $\mathcal{L}(X)$ for the space $\mathcal{L}\left(N(X), v_{0}, A m p(X)\right)$ introduced in Definition 2.13.

2.1. The Kähler period domain. Let $N$ be a non-degenerate lattice of signature $(2, \rho)$.

Definition 2.2. We define the Kähler period domain to be

$$
\mathfrak{D}(N)=\left\{[z] \in \mathbb{P}\left(N_{\mathbb{C}}\right) \mid z^{2}=0, z . \bar{z}>0\right\} \subset \mathbb{P}\left(N_{\mathbb{C}}\right)
$$

We also introduce the following open subset of $N_{\mathbb{C}}$ :

$$
\mathcal{P}(N)=\left\{z \in N_{\mathbb{C}} \mid \mathbb{R}\langle\operatorname{Re}(z), \operatorname{Im}(z)\rangle \subset N_{\mathbb{R}} \text { is a positive 2-plane }\right\} \subset N_{\mathbb{C}} .
$$

This set carries a natural free $G l_{2}(\mathbb{R})$-action by identifying $N_{\mathbb{C}}=N \otimes_{\mathbb{Z}} \mathbb{C}$ with $N \otimes_{\mathbb{Z}} \mathbb{R}^{2}$.

Lemma 2.3. There is a canonical map

$$
\theta: \mathcal{P}(N) \longrightarrow \mathfrak{D}(N)
$$

which is a principal $G l_{2}^{+}(\mathbb{R})_{2}^{+}$-bundle.

Proof. This map is most easily described using the canonical isomorphism $\mathfrak{D}(N) \cong G r_{2}^{p o}\left(N_{\mathbb{R}}\right)$, where $G r_{2}^{p o}\left(N_{\mathbb{R}}\right)$ is the Grassmann manifold of positive definite, oriented two-planes in $N_{\mathbb{R}}$ (cf. [BBD85, VII. Lem.1]). We define $\theta$ to map a vector $[z] \in \mathcal{P}(N)$ to the oriented two-plane $P=\mathbb{R}\langle\operatorname{Re}(z), \operatorname{Im}(z)\rangle$. As $G l_{2}^{+}(\mathbb{R})$ acts simply and transitively on the set of oriented bases of $P$, this map is a principal $G l_{2}^{+}(\mathbb{R})$-bundle.

In the case $N=N(X)=H^{0}(X) \oplus N S(X) \oplus H^{4}(X)$ there is a well known tube model of the period domain, given by

$$
\exp :\left\{z=x+i y \in N S(X)_{\mathbb{C}} \mid y^{2}>0\right\} \stackrel{\cong}{\longrightarrow} \mathfrak{D}(N), z \mapsto\left[\left(1, z, \frac{1}{2} z^{2}\right)\right] .
$$

To define this map we used the full information about the embedding $U \cong$ $H^{0}(X) \oplus H^{4}(X)$ into $N(X)$. In this section we will construct a similar map, which only depends on the isotropic vector $v_{0}=(0,0,1)$. Compare also Dol96, Sec. 4].

Let $N$ be a non-degenerate lattice of signature $(2, \rho), \rho \geq 1$. To a primitive isotropic vector $v \in N(X)$ we associate the lattice

$$
L(v)=v^{\perp} / \mathbb{Z} v=\{z \in N \mid z . v=0\} / \mathbb{Z} v
$$

of signature $(1, \rho-1)$ and the affine space $A(v)=\{z \in N \mid z \cdot v=-1\} / \mathbb{Z} v$ over $L(v)$. Note that, if $N=N(X)$ and $v=v_{0}$, then $L(v) \cong N S(X)$.

Definition 2.4. We define the tube domain associated to $N$ and $v$ as

$$
T(N, v)=A(v)_{\mathbb{R}} \times C(L(v))
$$

where $C(L(v))=\left\{y \in L(v)_{\mathbb{R}} \mid y^{2}>0\right\}$. Note that $A(v)_{\mathbb{R}}$ is naturally an affine space over $L(v)_{\mathbb{R}}$. We consider $T(N, v)$ as a subset of $N_{\mathbb{C}} / \mathbb{C} v$ by mapping $(x, y)$ to $x+i y \in N_{\mathbb{C}} / \mathbb{C} v$. We will often write $x+i y$ for a pair $(x, y) \in T(N, v)$. 
Lemma 2.5. There is a canonical map $\operatorname{Exp}_{v}: T(N, v) \rightarrow \mathcal{P}(N)$ such that

$$
\exp _{v}=\theta \circ \operatorname{Exp}_{v}: T(N, v) \longrightarrow \mathfrak{D}(N)
$$

is an isomorphism.

Proof. We construct the inverse to $\operatorname{Exp}_{v}$. The set

$$
Q(v)=\left\{z \in N_{\mathbb{C}} \mid z^{2}=0, z \cdot \bar{z}>0, z \cdot v=-1\right\} \subset \mathcal{P}(N)
$$

is a section for the $G l_{2}^{+}(\mathbb{R})$-action on $\mathcal{P}(N)$.

One checks immediately, that the projection $N_{\mathbb{C}} \rightarrow N_{\mathbb{C}} / \mathbb{C} v$ induces an isomorphism $Q(v) \rightarrow T(N, v)$. Define $\operatorname{Exp}_{v}$ to be the inverse of this isomorphism.

Remark 2.6. In particular, we obtain a section of the $G l_{2}^{+}(\mathbb{R})$-bundle $\theta$, namely $q_{v}=\operatorname{Exp}_{v} \circ \exp _{v}^{-1}: \mathfrak{D}(N) \rightarrow \mathcal{P}(N)$.

Lemma 2.7. Let $g \in O(N)$ be an isometry of $N$, then $g$ induces a commutative diagram

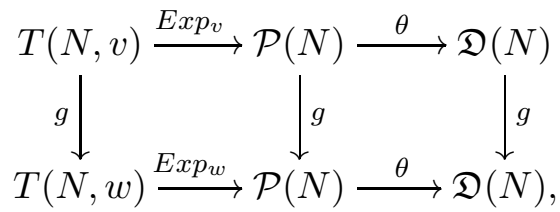

where $w=g \cdot v$.

2.2. Roots, Walls and Chambers. Recall that, every lattice $N$ determines a root-system $\Delta(N)=\left\{\delta \in N \mid \delta^{2}=-2\right\}$. To every root $\delta \in \Delta(N)$ there is an associated reflection, $s_{\delta}: w \mapsto w+(\delta . w) \delta$ which is an involutive isometry. The subgroup $W(N) \subset O(N)$ generated by the reflections is called Weyl group.

If we are given an isotropic vector $v \in N$ we define

$\Delta^{>0}(N, v)=\{\delta \in \Delta(N) \mid-v . \delta>0\}, \quad \Delta^{0}(N, v)=\{\delta \in \Delta(N) \mid v . \delta=0\}$.

The group generated by the reflections $\left\{s_{\delta} \mid \delta \in \Delta^{0}(N, v)\right\}$ is denoted by $W^{0}(N, v)$.

Definition 2.8. To $\delta \in \Delta(N)$ we associate a divisor

$$
D(\delta)=\{[z] \mid z . \delta=0\} \subset \mathfrak{D}(N)
$$

and define $\mathfrak{D}_{0}(N)=\mathfrak{D}(N) \backslash \bigcup\{D(\delta) \mid \delta \in \Delta(N)\}$.

The connected components of $\mathfrak{D}(N) \cong T(N, v)$ are clearly contractible. In contrast the space $\mathfrak{D}_{0}(N)$ is the complement of a infinite number of hypersurfaces and will therefore in general not even have a finitely generated fundamental group. Following Bridgeland (cf. Remark 4.10) we will decompose $\mathfrak{D}_{0}(N)$ into a union of codimension-one submanifolds called walls and their complements called chambers such that each individual chamber is contractible.

Definition 2.9. Given $\delta \in \Delta^{>0}(N, v)$ and a primitive, isotropic vector $v \in N$, we define a real, codimension one submanifold, called wall

$$
W_{A}(\delta, v)=\left\{[z] \in \mathfrak{D}(N) \mid-z . \delta / z . v \in \mathbb{R}_{\leq 0}\right\} \subset \mathfrak{D}(N) .
$$


To a vector $\delta \in \Delta^{0}(N, v)$ we associate the wall

$$
W_{C}(\delta, v)=\{[z] \in \mathfrak{D}(N) \mid-z . \delta / z . v \in \mathbb{R}\} .
$$

One can check, that $W_{C}(\delta, v)$ only depends on the image $l$ of $\delta$ in $L(v)=$ $v^{\perp} / \mathbb{Z} v$. Therefore we write also $W_{C}(l, v)$ for $W_{C}(\delta, v)$. We define

$$
\begin{aligned}
& \mathfrak{D}_{A}(N, v)=\mathfrak{D}(N) \backslash \bigcup\left\{W_{A}(\delta, v) \mid \delta \in \Delta^{>0}(N, v)\right\} \\
& \mathfrak{D}_{C}(N, v)=\mathfrak{D}(N) \backslash \bigcup\left\{W_{C}(\delta, v) \mid \delta \in \Delta^{0}(N, v)\right\} .
\end{aligned}
$$

We denote the intersections $\mathfrak{D}_{0}(N) \cap \mathfrak{D}_{A}(N, v), \mathfrak{D}_{A}(N, v) \cap \mathfrak{D}_{C}(N, v)$, etc. by $\mathfrak{D}_{0, A}(N, v), \mathfrak{D}_{A, C}(N, v)$, etc., respectively. For any combination $*$ of the symbols $0, A, C$ we set

$$
T_{*}(N, v)=\exp _{v}^{-1}\left(\mathfrak{D}_{*}(N, v)\right) .
$$

Remark 2.10. The seemingly unnatural notation, $-z . \delta / z . v \in \mathbb{R}_{\leq 0}$, is chosen since for $z=\operatorname{Exp}_{v}(x+i y)$ we have $z \cdot v=-1$, and hence $-z . \delta / z \cdot v=z . \delta$.

The sets considered above are indeed complex manifolds as the unions of $D(\delta), W_{A}(\delta, v)$ and $W_{C}(l, v)$ are locally finite [Bri08, Lem. 11.1].

If $\delta \in \Delta^{0}(N, v)$, then $D(\delta) \subset W_{C}(\delta, v)$, and if $\delta \in \pm \Delta^{>0}(N, v)$, then $D(\delta) \subset W_{A}(\delta, v)$. Therefore,

$$
\mathfrak{D}_{A, C}(N, v) \subset \mathfrak{D}_{0}(N, v) .
$$

Lemma 2.11. Bri08, Lem. 6.2, Lem. 11.1] Let

$$
\mathfrak{D}_{>2}(N, v)=\left\{\exp _{v}(x+i y) \in \mathfrak{D}(N) \mid y^{2}>2\right\} \subset \mathfrak{D}(N)
$$

and denote by $\mathfrak{D}_{0,>2}(N, v)=\mathfrak{D}_{>2}(N, v) \cap \mathfrak{D}_{0}(N, v)$ etc. the various intersections.

Then

$$
\mathfrak{D}_{>2}(N, v) \subset \mathfrak{D}_{A}(N, v)
$$

and the inclusions $\mathfrak{D}_{>2}(N, v) \subset \mathfrak{D}_{A}(N, v), \mathfrak{D}_{0,>2}(N, v) \subset \mathfrak{D}_{0, A}(N, v)$ and $\mathfrak{D}_{0, C,>2}(N, v) \subset \mathfrak{D}_{0, C, A}(N, v)$ are deformation retracts.

Recall that, $L=L(v)$ is a lattice of signature $(1, \rho-1)$. We defined $C(L)=\left\{y \in L_{\mathbb{R}} \mid y^{2}>0\right\}$ to be the positive cone. This space has two connected components, let $C(L)^{+}$be one of them. For $l \in \Delta(L)$ define a wall $W(l)=\{y \in C(L) \mid y . l=0\}$ and set $C(L)_{0}=C(L) \backslash \bigcup\{W(l) \mid l \in \Delta(L)\}$. Connected components of $C(L)_{0}$ are called chambers.

Lemma 2.12. We have

$$
T_{C}(N, v)=A(v)_{\mathbb{R}} \times C_{0}(L(v)) .
$$

Moreover, the connected components of $\mathfrak{D}_{0, A, C}(N, v) \subset \mathfrak{D}_{0}(N)$ are contractible.

Proof. The first assertion is a direct calculation. It follows, that the connected components of $\mathfrak{D}_{C}(N, v)$ and $\mathfrak{D}_{C,>2}(N, v)$ are contractible. Now, the components of $\mathfrak{D}_{0, A, C}(N, v)$ are contractible since Lemma 2.11 shows that $\mathfrak{D}_{C,>2}(N, v) \subset \mathfrak{D}_{0, A, C}(N, v)$ is a deformation retract. 
Definition 2.13. If we are given a chamber $A m p \subset C(L(v))_{0}$ we define $\mathcal{L}(N, v, A m p) \subset \mathfrak{D}_{0, A, C}(N, v)$ to be the connected component containing the vectors $\exp _{v}(x+i y)$ with $y \in A m p, y^{2}>2$.

The connected component of $\mathfrak{D}(N)$ containing $\mathcal{L}(N, v, A m p)$ is denoted by $\mathfrak{D}^{+}(N)$. We introduce also the notation $\mathfrak{D}_{*}^{+}(N)=\mathfrak{D}^{+}(N) \cap \mathfrak{D}_{*}(N, v)$ for a combination $*$ of the symbols $0, A, C,>2$.

The orthogonal group $O(N)$ acts on $\mathfrak{D}(N)$. We denote by $O^{+}(N)$ be the index two subgroup preserving the connected components of $\mathfrak{D}(N)$.

Remark 2.14. The set $\mathcal{L}(N, v, A m p)$ can be described more explicitly as $\{\exp (x+i y) \in \mathfrak{D}(X) \mid y \in A m p(X),(*)\}$ where $(*)$ is the condition

$$
\operatorname{Exp}(x+i y) . \delta \notin \mathbb{R}_{\leq 0} \quad \text { for all } \delta \in \Delta^{>0}(N(X), v) .
$$

This is the description used in Bri08.

\section{MA's THEOREM}

The goal of this section is to explain Ma's theorem about cusps of the Kähler moduli space of a K3 surface ([Ma09], Ma10]).

We use the recent result HMS09 to make the construction of the Kähler moduli space intrinsic to the derived category. This allows us to formulate Ma's theorem in a more symmetric way.

3.1. The Kähler moduli space. Recall from Definition 2.13, that $\mathfrak{D}(X)=$ $\mathfrak{D}(N(X))$ has a distinguished connected component $\mathfrak{D}^{+}(X)$ containing the vectors $\exp (x+i y)$ with $y \in N S(X)$ ample. The key ingredient for our construction of $K M(\mathcal{T})$ is the following theorem.

Theorem 3.1. HLOY04, Plo05, [HMS09, Cor. 4.10] The image of

$$
\operatorname{Aut}\left(\mathcal{D}^{b}(X)\right) \longrightarrow O_{\text {Hodge }}(\tilde{H}(X, \mathbb{Z}))
$$

is the index-two subgroup $O_{\text {Hodge }}^{+}(\tilde{H}(X, \mathbb{Z}))$ of isometries preserving the component $\mathfrak{D}^{+}(X) \subset \mathfrak{D}(X)$.

Let $\Phi: \mathcal{D}^{b}(X) \rightarrow \mathcal{D}^{b}(Y)$ be a derived equivalence between two K3 surfaces. Then the isomorphism $\Phi^{H}: \mathfrak{D}(X) \rightarrow \mathfrak{D}(Y)$ maps $\mathfrak{D}^{+}(X)$ to $\mathfrak{D}^{+}(Y)$.

This theorem allows us to make the following definition.

Definition 3.2. Let $\mathfrak{D}^{+}(\mathcal{T})$ be the connected component of the period domain $\mathfrak{D}(\mathcal{T})=\mathfrak{D}(N(\mathcal{T}))$ which is mapped to $\mathfrak{D}^{+}(X)$ under every derived equivalence $\mathcal{T} \cong \mathcal{D}^{b}(X)$.

We define the Kähler moduli space of $\mathcal{T}$ to be

$$
K M(\mathcal{T})=\Gamma_{\mathcal{T}} \backslash \mathfrak{D}^{+}(\mathcal{T})
$$

where $\Gamma_{\mathcal{T}}$ is the image of $\operatorname{Aut}(\mathcal{T})$ in $O(N(\mathcal{T}))$.

Remark 3.3. Let us introduce the notation $K M(X)$ for $K M\left(\mathcal{D}^{b}(X)\right)$. Theorem 3.1 shows, that we have a canonical isomorphism

$$
K M(X) \cong \Gamma_{X}^{+} \backslash \mathfrak{D}^{+}(X),
$$

where $\Gamma_{X}^{+} \subset O(N(X))$ is the image of $O_{\text {Hodge }}^{+}(\tilde{H}(X, \mathbb{Z}))$ in $O(N(X))$. Ma works in the setting $\mathcal{T}=\mathcal{D}^{b}(X)$ and uses $\Gamma_{X}^{+} \backslash \mathfrak{D}^{+}(X)$ as definition for the Kähler moduli space. 
Remark 3.4. There is another construction of the Kähler moduli space using the theory of Bridgeland stability conditions which is proved in the appendix cf. Corollary 7.10 :

$$
K M_{0}(X) \cong A u t^{\dagger}\left(\mathcal{D}^{b}(X)\right) \backslash \operatorname{Stab}^{\dagger}(X) / \widetilde{G l} l_{2}^{+}(\mathbb{R}) .
$$

Here $K M_{0}(X)=\Gamma_{X}^{+} \backslash \mathfrak{D}_{0}^{+}(X) \subset K M(X)$ is the complement of a divisor and $A u t^{\dagger}\left(\mathcal{D}^{b}(X)\right)$ is the group of auto-equivalences respecting the distinguished component $\operatorname{Stab}^{\dagger}(X)$ of the stability manifold. This was also stated in Ma09 without proof.

Note that, this description is not intrinsic to the derived category as the component $\operatorname{Stab}^{\dagger}(X) \subset \operatorname{Stab}\left(\mathcal{D}^{b}(X)\right)$ may a priori depend on $X$. But in fact, no other component of the stability manifold is known.

Example 3.5. If $X$ has Picard rank $\rho(X)=1$ and the ample generator $H \in$ $N S(X)$ has square $H . H=2 n$, then the Kähler moduli space is isomorphic to a Fricke modular curve $K M(X) \cong \Gamma_{0}^{+}(n) \backslash \mathbb{H}$. See [Ma10, Sec. 5], Dol96, Thm. 7.1].

The subgroup $\Gamma_{\mathcal{T}} \subset O(N(\mathcal{T}))$ is of finite index since it contains

$$
O_{0}^{+}(N(\mathcal{T}))=O^{+}(N(\mathcal{T})) \cap K \operatorname{Ker}(O(N(\mathcal{T})) \rightarrow \operatorname{Aut}(A(N(\mathcal{T}))))
$$

where $A(N(\mathcal{T}))=N(\mathcal{T})^{\vee} / N(\mathcal{T})$ is the discriminant group, cf. [Ma09, Def. 3.1.]. Hence we can apply a general construction of Baily and Borel to compactify the Kähler moduli space.

Theorem 3.6 (Baily-Borel). [BB66] There is a natural compactification $K M(\mathcal{T}) \subset \overline{K M}(\mathcal{T})$ which is a normal, projective variety over $\mathbb{C}$.

The boundary $\partial K M(\mathcal{T})=\overline{K M}(\mathcal{T}) \backslash K M(\mathcal{T})$ consists of zero- and onedimensional components called cusps, which are in bijection to $\Gamma_{\mathcal{T}} \backslash \mathcal{B}_{i}$, where

$$
\mathcal{B}_{i}=\{I \subset N(\mathcal{T}) \mid I \text { primitive, isotropic, } r k(I)=i+1\}
$$

for $i=0,1$ respectively.

Definition 3.7. The set of zero-dimensional cusps is divided further with respect to divisibility. For $I \in \mathcal{B}_{0}$ we define

$$
\operatorname{div}(I)=\operatorname{g.c.d}(\{v \cdot w \mid v \in I, w \in N(\mathcal{T})\})
$$

and set $\mathcal{B}_{0}^{d}=\left\{I \in \mathcal{B}_{0} \mid \operatorname{div}(I)=d\right\}$. Cusps corresponding to elements of $\mathcal{B}_{0}^{1}$ are called standard cusps.

We call $v \in N$ a standard vector 1 if $v \cdot v=0$ and $\operatorname{div}(v):=\operatorname{div}(\mathbb{Z} v)=1$.

Remark 3.8. The group $\Gamma_{\mathcal{T}}$ contains the element $-i d_{N(\mathcal{T})}=[1]^{H}$ which interchanges the generators of any $I \in \mathcal{B}_{0}^{1}$. Therefore, the map $v \mapsto \mathbb{Z} v$ induces a bijection

$$
\Gamma_{\mathcal{T}} \backslash\{v \in N(\mathcal{T}) \mid v \text { standard }\} \cong\{\text { standard cusps of } \overline{K M}(\mathcal{T})\} .
$$

We will refer to standard cusps as equivalence classes $[v]=\Gamma_{\mathcal{T}} \cdot v$ via this bijection.

\footnotetext{
${ }^{1}$ This definition is not standard.
} 


\subsection{Ma's theorem.}

Definition 3.9. The Kähler moduli space of $\mathcal{D}^{b}(X)$ comes with a distinguished standard cusp $\left[v_{0}\right] \in \overline{K M}(X), v_{0}=(0,0,1) \in N(X)$, which is called large volume limit.

We are now ready to state Ma's theorem.

Theorem 3.10 (Ma). Ma09, Ma10] There is a canonical bijection

$$
\left\{X \text { K3 surface } \mid \mathcal{T} \cong \mathcal{D}^{b}(X)\right\}_{/ \cong} \longleftrightarrow\{\text { standard cusps of } \overline{K M}(\mathcal{T})\}
$$

The cusp of $\overline{K M}(\mathcal{T})$ associated to $Y$ corresponds to the large volume limit of $X$ under the isomorphism $\overline{K M}(\mathcal{T}) \cong \overline{K M}(X)$ induced by any equivalence $\mathcal{T} \cong \mathcal{D}^{b}(X)$.

We denote the K3 surface associated to a cusp $[v]$ by $X(v)$.

Proof. We sketch Ma's original proof for the case $\mathcal{T}=\mathcal{D}^{b}(X)$ and then generalize to our situation.

Every derived equivalence $\Phi: \mathcal{D}^{b}(Y) \rightarrow \mathcal{D}^{b}(X)$ induces an isometry $\Phi^{H}$ : $N(Y) \rightarrow N(X)$, and therefore an embedding of the hyperbolic plane

$$
U \cong H^{0}(Y) \oplus H^{4}(Y) \subset N(Y) \stackrel{\Phi^{H}}{\longrightarrow} N(X) .
$$

It follows from Orlov's derived global Torelli theorem Huy06, Prop. 10.10] that this construction induces a bijection

$$
\left\{Y \mathrm{~K} 3 \text { surface } \mid \mathcal{D}^{b}(X) \cong \mathcal{D}^{b}(Y)\right\}_{/ \cong} \longrightarrow \operatorname{Emb}(U, N(X)) / \Gamma_{X},
$$

where $\operatorname{Emb}(U, N(X))$ is the set of all embeddings of the hyperbolic plane $U$ into $N(X)$, and $\Gamma_{X} \subset O(N(X))$ is the image of $O_{\text {Hodge }}(\tilde{H}(X, \mathbb{Z}))$ in $O(N(X))$. The key insight of Ma is that the map $\varphi \mapsto \varphi(f)$, where $e, f \in U$ is the standard basis, induces a bijection

$$
\operatorname{Emb}(U, N(X)) / \Gamma_{X} \longrightarrow\{v \in N(X) \mid v \text { standard }\} / \Gamma_{X}^{+} .
$$

Combining with Remark 3.8 one gets a bijection

$$
\left\{Y \text { K3 surface } \mid \mathcal{D}^{b}(X) \cong \mathcal{D}^{b}(Y)\right\}_{/ \cong} \longrightarrow\{\text { standard cusps of } \overline{K M}(X)\}
$$

which maps $X$ maps to $\left[v_{0}\right]$. Note that, the Hodge structure $H^{2}(Y, \mathbb{Z})$ of a K3 surface $Y$ can be reconstructed from the associated cusp $[v]$ as the subquotient $v^{\perp} / v$ of $\tilde{H}(X, \mathbb{Z})$.

To generalize to arbitrary $\mathcal{T}$ we choose an equivalence $\mathcal{T} \cong \mathcal{D}^{b}(X)$ and claim that the above bijection is independent of this choice. Indeed, if we are given another equivalence $\mathcal{T} \cong \mathcal{D}^{b}(Y)$, then the composition $\Phi: \mathcal{D}^{b}(X) \cong$ $\mathcal{T} \cong \mathcal{D}^{b}(Y)$ induces a Hodge isometry $\Phi^{H}: \tilde{H}(X, \mathbb{Z}) \rightarrow \tilde{H}(Y, \mathbb{Z})$. If a standard vector $v \in N(\mathcal{T})$ corresponds to $v_{1} \in N(X)$ and $v_{2} \in N(Y)$ then $\Phi^{H}$ induces an isomorphism of Hodge structures

$$
H^{2}\left(X\left(v_{1}\right), \mathbb{Z}\right) \cong v_{1}^{\perp} / v_{1} \longrightarrow v_{2}^{\perp} / v_{2} \cong H^{2}\left(X\left(v_{2}\right), \mathbb{Z}\right)
$$

Now, the global Torelli theorem shows that the K3 surfaces $X\left(v_{1}\right)$ and $X\left(v_{2}\right)$ are isomorphic. 
Remark 3.11. Let $v \in N(X)$ be a standard vector defining a standard cusp of $\overline{K M}(X)$. The Fourier-Mukai partner $Y=X(v)$ associated to this cusp via Ma's theorem is determined up to isomorphism, by the property that

$$
H^{2}(X(v), \mathbb{Z}) \cong v^{\perp} / v
$$

as subquotient of $\tilde{H}(X, \mathbb{Z})$.

We cannot formulate an analogues statement for the cusps of $\overline{K M}(\mathcal{T})$ since there is no construction of the Hodge structure $\tilde{H}(X, \mathbb{Z})$ known, which is intrinsic to the category $\mathcal{D}^{b}(X)$.

Remark 3.12. Let $X$ be a K3 surface, $[v] \in \overline{K M}(X)$ a standard cusp and let $Y=X(v)$ be the associated Fourier-Mukai partner. We have seen that every derived equivalence $\Phi: \mathcal{D}^{b}(X) \stackrel{\sim}{\longrightarrow} \mathcal{D}^{b}(Y)$ maps $[v]$ to the large volume limit $\left[v_{0}\right] \in \overline{K M}(Y)$.

In appendix 7.4 we will strengthen this result in two directions. Firstly, we will construct a $\Phi$, with the property that $\Phi^{H}$ maps $v$ to $v_{0}$ and not only the orbit $[v]$ to $\left[v_{0}\right]$. Secondly, the equivalence $\Phi$ respects the distinguished component of the stability manifold (cf. Subsection 4.3).

\section{Stability CONDitions}

Our next goal is to relate the Kähler moduli space to the stability manifold. In this section we recall from [Bri07] and Bri08 the basic theory of Bridgeland stability conditions in the special case of a K3 surface. On the way we introduce the notation and establish some geometric results that will be used in sequel. The link to the Kähler moduli space will be made in section 5 and Corollary 7.10 in the appendix.

4.1. Definition of stability conditions. Let $X$ be a K3 surface. Recall from [Bri07, Def. 5.7, Def. 2.3., Prop. 5.3], that a stability condition $\sigma$ on $\mathcal{D}^{b}(X)$ consists of

(1) a heart $\mathcal{A}$ of a bounded t-structure on $\mathcal{D}^{b}(X)$ and

(2) a vector $z \in N(X)_{\mathbb{C}}$ called central charge

with the property that $Z: K(\mathcal{A}) \rightarrow \mathbb{C}, A \mapsto v(A) . z$ satisfies

$$
Z(A) \in \mathbb{H} \cup \mathbb{R}_{<0} \text { for all } A \in \mathcal{A}, A \neq 0 .
$$

We require moreover local-finiteness and the existence of Harder-Narasimhan filtrations.

In the usual definition, the datum of the heart is replaced by a collection of subcategories $\mathcal{P}(\phi) \subset \mathcal{A}, \phi \in \mathbb{R}$, called slicing. The equivalence to the above definition was shown in [Bri07, Prop. 5.3].

The main result about the stability manifold of a K3 surface is the following theorem.

Theorem 4.1. Bri07, Cor. 1.3], Bri08, Thm. 1.1] The set of all stability conditions on a K3 surface $X$ has the structure of a (finite-dimensional) complex manifold Stab $(X)$.

There is a distinguished connected component $\operatorname{Stab}^{\dagger}(X)$ of $\operatorname{Stab}(X)$ such that the map $\sigma=(\mathcal{A}, z) \mapsto z$ induces a Galois cover

$$
\pi: \operatorname{Stab}^{\dagger}(X) \longrightarrow \mathcal{P}_{0}^{+}(X) .
$$


Moreover, the Galois group is identified with the $A u t_{0}^{\dagger}\left(\mathcal{D}^{b}(X)\right) \subset \operatorname{Aut}\left(\mathcal{D}^{b}(X)\right)$, the group of auto-equivalences that respect the component $\operatorname{Stab}^{\dagger}(X)$ and act trivially on the cohomology $\tilde{H}(X, \mathbb{Z})$.

4.2. Group actions. Given a derived equivalence $\Phi: \mathcal{D}^{b}(X) \stackrel{\sim}{\longrightarrow} \mathcal{D}^{b}(Y)$ and a stability condition $(\mathcal{A}, z)$ on $\mathcal{D}^{b}(X)$ we get an induced stability condition $\Phi_{*}(\mathcal{A}, z)=\left(\Phi(\mathcal{A}), \Phi^{H}(z)\right)$ on $\mathcal{D}^{b}(Y)$. In this way we obtain a left action of the group $\operatorname{Aut}\left(\mathcal{D}^{b}(X)\right)$ on $\operatorname{Stab}(X)$.

There is also a right action of the group

$\widetilde{G l} l_{2}^{+}(\mathbb{R})=\left\{(T, f) \mid T \in G l_{2}^{+}(\mathbb{R}), f: \mathbb{R} \rightarrow \mathbb{R}, f(\phi+1)=f(\phi)+1\right.$ with $\left.(\#)\right\}$ on $\operatorname{Stab}(X)$ (cf. [Bri07, Lem. 8.2.]). Here (\#) stands for the condition $\mathbb{R}_{>0} T \cdot \exp (i \pi \phi)=\mathbb{R}_{>0} \exp (i \pi f(\phi))$.

Example 4.2. For $\lambda \in \mathbb{R}$ set $\Sigma_{\lambda}=(\exp (i \pi \lambda), \phi \mapsto \phi+\lambda) \in \widetilde{G l}_{2}^{+}(\mathbb{R})$. Then the action of the shift [1] equals the action of the $\Sigma_{1}$ on the stability manifold.

4.3. Construction of stability conditions. Explicit examples of stability conditions on a K3 surface are constructed as follows.

Fix classes $\beta \in N S(X)_{\mathbb{R}}$ and $\omega \in A m p(X)$ and define a central charge

$$
\operatorname{Exp}(\beta+i \omega)=\left(1, \beta+i \omega, \frac{1}{2}(\beta+i \omega)^{2}\right) \in N(X)_{\mathbb{C}} .
$$

For a torsion free sheaf $A$ of positive rank denote by

$$
\begin{aligned}
& \mu_{\omega}^{\min }(A)=\inf \left\{\mu_{\omega}(Q) \mid A \rightarrow Q, Q \text { torsion free }\right\} \\
& \mu_{\omega}^{\max }(A)=\sup \left\{\mu_{\omega}(S) \mid S \hookrightarrow A\right\}
\end{aligned}
$$

the extremal slopes. Define full subcategories of $\operatorname{Coh}(X)$ by

$$
\begin{aligned}
& \mathcal{T}=\left\{A \in C \operatorname{Coh}(X) \mid A \text { torsion or } \mu_{\omega}^{\min }\left(A / A_{\text {tors }}\right)>\beta . \omega\right\} \\
& \mathcal{F}=\left\{A \in \operatorname{Coh}(X) \mid A \text { torsion free and } \mu_{\omega}^{\max }(A) \leq \beta . \omega\right\} .
\end{aligned}
$$

The following full subcategory of $\mathcal{D}^{b}(X)$ is a heart of a bounded t-structure. $\mathcal{A}(\beta, \omega)=\left\{E \in \mathcal{D}^{b}(X) \mid H^{0}(E) \in \mathcal{T}, H^{-1}(E) \in \mathcal{F}, H^{i}(E)=0\right.$ if $\left.i \neq 0,-1\right\}$.

Theorem 4.3. Bri08, Lem. 6.2, Prop. 11.2] The pair

$$
\sigma(\beta, \omega)=(\mathcal{A}(\beta, \omega), z=\operatorname{Exp}(\beta+i \omega))
$$

is a stability condition on $\mathcal{D}^{b}(X)$ if $\theta(z)=\exp (\beta+i \omega) \in \mathcal{L}(X) \subset \mathfrak{D}(X)$.

The set of all stability conditions arising in this way is denoted by $V(X)$.

The connected component of $\operatorname{Stab}(X)$ containing $V(X)$ is called distinguished component and denoted by $\operatorname{Stab}^{\dagger}(X)$.

Let $\Phi: \mathcal{D}^{b}(X) \rightarrow \mathcal{D}^{b}(Y)$ be a derived equivalence between two K3 surfaces. We say $\Phi$ respects the distinguished component if $\Phi_{*} \operatorname{Stab}^{\dagger}(X)=$ $\operatorname{Stab}^{\dagger}(Y)$.

Remark 4.4. The heart $\mathcal{A}(\beta, \omega \lambda)$ is independent of $\lambda>0$. Indeed, we have $\mu_{\lambda \omega}(A)=\lambda \mu_{\omega}$ and hence the conditions $\mu_{\omega}^{\min }\left(A / A_{\text {tors }}\right)>\beta . \omega$ and $\mu_{\omega}^{\max }\left(A / A_{\text {tors }}\right) \leq \beta . \omega$ are invariant under $\omega \mapsto \lambda \omega$. Therefore $\mathcal{T}$ and $\mathcal{F}$ do not depend on $\lambda$. 
Remark 4.5. By [Bri08, Prop. 10.3] the action of $\widetilde{G l} l_{2}^{+}(\mathbb{R})$-action on $V(X)$ is free. We introduce the notation $U(X):=V(X) \cdot \widetilde{G l} l_{2}^{+}(\mathbb{R}) \cong V(X) \times \widetilde{G l} l_{2}^{+}(\mathbb{R})$ for the image.

The following proposition gives an important characterization of $U(X)$.

Proposition 4.6. Bri08, Def. 10.2, Prop. 10.3] Let $\sigma=(\mathcal{A}, z)$ be a stability condition on $\mathcal{D}^{b}(X)$. Then $\sigma \in U(X)$ if and only if the following properties hold.

(1) All skyscraper sheaves $\mathcal{O}_{x}$ are stable of the same phase.

(2) The vector $z$ lies in $\mathcal{P}_{0}(X)$.

\subsection{Geometric refinements.}

Remark 4.7. Let us summarize the above discussion in the following diagram:

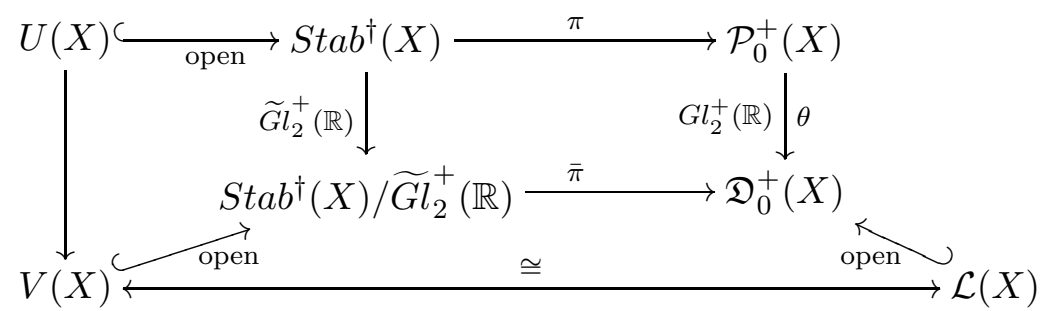

Here we identify $V(X)$ with its image in $\operatorname{Stab}^{\dagger}(X) / \widetilde{G l} l_{2}^{+}(\mathbb{R})$. The maps $\pi, \bar{\pi}$ are covering spaces. Moreover, the map $\pi: U(X) \rightarrow \theta^{-1}(\mathcal{L}(X))$ is a covering space with fiber $\mathbb{Z}$.

Lemma 4.8. Consider the map $\sigma: \mathcal{L}(X) \rightarrow V(X)$ which maps $\exp (\beta+i \omega)$ to the stability condition $\sigma(\beta, \omega)$.

Let $\overline{\mathcal{L}}_{0,>2}(X)$ be the closure of $\mathcal{L}_{0,>2}=\mathcal{L}(X) \cap \mathfrak{D}_{0,>2}^{+}(X)$ in $\mathfrak{D}_{0,>2}^{+}(X)$, and $\bar{V}_{>2}(X)$ be the intersection of $\bar{V}(X)$ with $\pi^{-1}\left(\mathfrak{D}_{>2}(X)\right)$.

Then there is a unique continuous extension of $\left.\sigma\right|_{\mathcal{L}_{0,>2}}(X)$ to an isomorphism

$$
\sigma^{*}: \overline{\mathcal{L}}_{0,>2}(X) \stackrel{\sim}{\longrightarrow} \bar{V}_{>2}(X) .
$$

Proof. Under the isomorphism exp $: T\left(N(X), v_{0}\right) \rightarrow \mathfrak{D}(X)$ the set $\overline{\mathcal{L}}_{0,>2}(X)$ gets identified with $\left\{x+i y \mid y \in \overline{A m p}(X), y^{2}>2,(*)\right\}$ where $(*)$ is the condition

$$
(x+i y) . l \notin \mathbb{Z} \quad \text { for all } \quad l \in \Delta(N S(X))
$$

As we have $y . l \geq 0$ for $y \in \overline{A m p}(X)$, we can retract $\overline{\mathcal{L}}_{0,>2}(X)$ into the subset $\mathcal{L}(X)_{>2}=\mathcal{L}(X) \cap \mathfrak{D}_{>2}$ via the homotopy $(x+i y, t) \mapsto x+i y+$ tiw for $t \in[0,1]$ and $\omega \in A m p(X)$. It follows that $\overline{\mathcal{L}}_{0,>2}(X)$ is contractible.

Hence, the restriction of the covering space $\pi: \operatorname{Stab}^{\dagger}(X) \rightarrow \mathcal{P}_{0}^{+}(X)$ to $q\left(\overline{\mathcal{L}}_{0,>2}(X)\right) \subset \mathcal{P}_{0}^{+}(X)$ is trivial (cf. Remark 2.6 for the definition of $q=q_{v_{0}}$ ) and there is a unique section $s$ extending $\sigma \circ \theta: q(\mathcal{L}(X)) \rightarrow V(X)$ to $q\left(\overline{\mathcal{L}}_{0,>2}(X)\right)$. We now set $\sigma^{*}=s \circ q$.

Next, we recall Bridgeland's description of the boundary of $U(X)$. As a well known consequence we get a covering of the stability manifold by certain translates of the closure $\bar{U}(X)$. 
Theorem 4.9. Bri08, Thm. 12.1.] The boundary $\partial U(X)$ is contained in a locally finite union of real codimension-one submanifolds. If $x \in \partial U(X)$ is a general boundary point, i.e. lies only on one of these submanifolds, then precisely one of the following possibilities hold.

$\left(A^{+}\right)$There is a rank $r$ spherical vector bundle $A$ such that the stable factors of the objects $\mathcal{O}_{x}, x \in X$ are $A$ and $T_{A}\left(\mathcal{O}_{x}\right)$.

$\left(A^{-}\right)$There is a rank $r$ spherical vector bundle $A$ such that the stable factors of the objects $\mathcal{O}_{x}, x \in X$ are $A[2]$ and $T_{A}^{-1}\left(\mathcal{O}_{x}\right)$.

$\left(C_{k}\right)$ There is a non-singular rational curve $C$ and an integer $k$ such that $\mathcal{O}_{x}$ is stable if and only if $x \notin C$. If $x \in C$, then it has a stable factor $\mathcal{O}_{C}(k)[1]$.

Remark 4.10. If $\sigma \in \bar{U}(X)$ satisfies condition $\left(A^{+}\right)$or $\left(A^{-}\right)$, then the centralcharges of $\mathcal{O}_{x}$ and $A$ are co-linear: $Z(A) / Z\left(v_{0}\right) \in \mathbb{R}_{>0}$. This is precisely the condition we used in Definition 2.9 to define the A-type wall $W_{A}\left(v(A), v_{0}\right) \subset$ $\mathfrak{D}(X)$. Therefore the image $\bar{\pi}(\sigma)$ of in $\mathfrak{D}(X)$ lies on $W_{A}\left(v(A), v_{0}\right)$.

Similarly, if $\sigma \in \bar{U}(X)$ satisfies condition $\left(C_{k}\right)$, then $\bar{\pi}(\sigma)$ lies on the wall $W_{C}\left(v\left(\mathcal{O}_{C}\right), v_{0}\right)$ of type $C$.

Definition 4.11. Let $\widetilde{W}(X) \subset \operatorname{Aut}\left(\mathcal{D}^{b}(X)\right)$ be the group generated by the spherical twists $T_{A}^{2}, T_{\mathcal{O}_{C}(k)}$ for all (-2)-curves $C, k \in \mathbb{Z}$ and spherical vector bundles $A$, which occur in the description of the boundary $\partial U(X)$ given in Theorem 4.9 .

Remark 4.12. One can check, that all equivalences $\Phi \in \widetilde{W}(X)$ have the property $\Phi^{H}\left(v_{0}\right)=v_{0}$. This means we get a map

$$
\widetilde{W}(X) \longrightarrow W^{0}\left(N(X), v_{0}\right) \subset O(N(X)), \quad \Phi \mapsto \Phi^{H} .
$$

As we will see in Proposition 7.7, this map is surjective.

Remark 4.13. All equivalences $\Phi \in \widetilde{W}(X)$ respect the distinguished component $\operatorname{Stab}^{\dagger}(X)$. Indeed, the spherical twists $T_{\mathcal{O}_{C}(k)}$ and $T_{A}^{2}$, which generate $\widetilde{W}(X)$, map the corresponding boundary components of $\partial U(X)$ into $\partial U(X)$, cf. [Bri08, Thm. 12.1.]. We will study equivalences with this property more closely in section 7 .

The following lemma is an easy consequence of the proof of Bri08, Prop. 13.2.].

Lemma 4.14. The translates of the closed subset $\bar{U}(X)$ under the group $\widetilde{W}(X) \operatorname{cover} \operatorname{Stab}^{\dagger}(X)$ :

$$
\bigcup_{\Phi \in \widetilde{W}(X)} \Phi_{*} \bar{U}(X)=\operatorname{Stab}^{\dagger}(X) .
$$

One can show, moreover, that the intersections of the interiors $U(X) \cap$ $\Phi_{*} U(X)$ are empty unless $\Phi=i d$. However, we will not need this refinement.

\footnotetext{
${ }^{2}$ Here $T_{A}$ denotes the spherical twist functor, cf. Huy06, Sec. 8.1.].
} 


\section{Cusps And hearts of Stability CONDitions}

In this section we proof our main geometric results about cusps and stability conditions. We will introduce the notion of a linear degeneration to a cusp in the Kähler moduli space and classify all paths in the stability manifold mapping to linear degenerations. Moreover, we construct paths in the stability manifold with special limiting hearts.

We will use the results proved in appendix 7 .

\subsection{Linear degenerations in $\overline{K M}(X)$.}

Definition 5.1. Let $\gamma(t) \in K M(X), t \gg 0$ be a path in the Kähler moduli space and $[v]$ a standard cusp of $\overline{K M}(X)$.

We say $\gamma(t)$ is a linear degeneration to a cusp $[v] \in \overline{K M}(X)$ if there exists a lift $\alpha(t)$ of $\gamma(t)$ to $\mathfrak{D}^{+}(X)$ and a vector $w \in \Gamma_{X} \cdot v$ such that

$$
\alpha(t)=\exp _{w}\left(x_{0}+i t y_{0}\right)
$$

for some $x_{0} \in A(w)_{\mathbb{R}}, y_{0} \in C(L(w))$.

Proposition 5.2. Let $\gamma(t)$ be a linear degeneration to $[v] \in \overline{K M}(X)$.

(1) The limit of $\gamma(t)$ in $\overline{K M}(X)$ is

$$
\lim _{t \rightarrow \infty} \gamma(t)=[v] \in \overline{K M}(X) .
$$

(2) If $\beta(t)$ is another lift of $\gamma(t)$ to $\mathfrak{D}^{+}(X)$, then there is a $g \in \Gamma_{X}$ such that

$$
\beta(t)=\exp _{w^{\prime}}\left(x_{0}^{\prime}+i t y_{0}^{\prime}\right)
$$

for $w^{\prime}=g \cdot w, x_{0}^{\prime}=g \cdot x_{0}, y_{0}^{\prime}=g \cdot y_{0}$ and $t \gg 0$.

Proof. In [Loo03, 2.2] Looijenga constructs a basis of neighborhoods of $[v] \in$ $K M(X)$ as follows. Let $\Gamma_{v}=\left\{g \in \Gamma_{X}^{+} \mid g \cdot v=v\right\}$. Consider the exponential parametrization

$$
\exp _{v}: A(v)_{\mathbb{R}} \times C^{+}(L(v)) \longrightarrow \mathfrak{D}^{+}(X) .
$$

The semi-group $L(v)_{\mathbb{R}} \times C^{+}(L(v))$ acts on $A(v)_{\mathbb{R}} \times C^{+}(L(v))$ by translation, and hence also on $\mathfrak{D}^{+}(X)$. For an open subset $K$ of $\mathfrak{D}^{+}(X)$ let

$$
U(K, v)=\Gamma_{v} \cdot\left(L(v)_{\mathbb{R}} \times C^{+}(L(v))\right) \cdot K \subset \mathfrak{D}^{+}(X),
$$

which is also an open subset of $\mathfrak{D}^{+}(X)$.

Then the images of $U(K, v)$ in $K M(X)=\Gamma_{X}^{+} \backslash \mathfrak{D}^{+}(X)$, where $K \subset \mathfrak{D}^{+}(X)$ runs through all open and non-empty subsets of $\mathfrak{D}^{+}(X)$, form a basis of neighborhoods of $[v] \in \overline{K M}(X)$.

It is easy to see that every linear degeneration $\gamma(t) \in K M(X)$ lies eventually in any of the subsets $U(K, v)$. This shows the first claim.

We now proceed to the second claim. By assumption, there are $g(t) \in \Gamma_{X}^{+}$ such that $\beta(t)=g(t) \cdot \alpha(t)$. We have to show that it is possible to choose $g(t)$ independent of $t$ for $t \gg 0$. Note that, the action of $\Gamma_{X}$ on $\mathfrak{D}(X)$ is not fixed point free, and hence the elements $g(t)$ itself may depend on $t$.

By [BB66, Thm. 4.9. iv)] there is a subbasis $\left\{U\left(K^{\prime}, v\right)\right\}$ of neighborhoods of the cusp $[v]$ such that $\Gamma_{v} \backslash U\left(K^{\prime}, v\right)$ injects into $K M(X)$.

Note that $\Gamma_{v}$ acts linearly on the tube model, i.e. $g \cdot \exp (x+i y)=$ $\exp (g \cdot x+i g \cdot y)$ for the canonical action of $\Gamma_{v}$ on $A(v)$ and $L(v)$. Therefore 
the fixed point locus of an element $g \in \Gamma_{v}$ is either disjoint form $\alpha(t)$ or contains $\alpha(t)$ for all $t$.

This can be used to construct a uniform $g=g(t)$. Indeed, let $t_{0} \gg 0$ and suppose, without loss of generality, that $\alpha\left(t_{0}\right)=\beta\left(t_{0}\right)$. For $g \in \Gamma_{v}$ the closed sets

$$
C(g)=\left\{t \in[0,1] \mid g \cdot \alpha\left(t_{0}+t\right)=\beta\left(t_{0}+t\right)\right\}
$$

cover the interval $[0,1]$. Our argument above shows that if $C(g) \cap C\left(g^{\prime}\right) \neq \emptyset$, then $C(g)=C\left(g^{\prime}\right)$. By the properness of the action we see that $C(g) \neq \emptyset$ only for finitely many $g$. But an interval cannot be covered non-trivially by finitely many disjoint closed subsets. If follows that $C(\mathrm{id})=\mathbb{R}_{\geq t_{0}}$.

5.2. Linear degenerations of stability conditions. We have the following natural maps from the stability manifold to the Kähler moduli space.

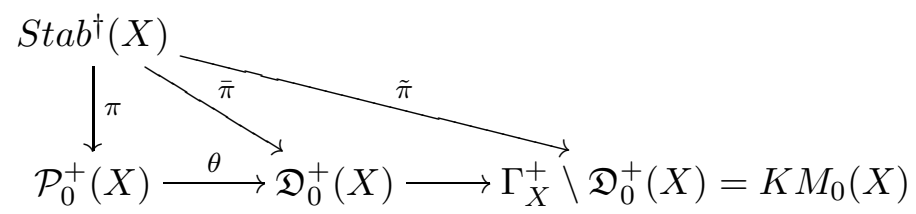

The goal of this section is to proof the following Theorem.

Theorem 5.3. Let $[v] \in \overline{K M}(X)$ be a standard cusp and $\sigma(t) \in \operatorname{Stab}^{\dagger}(X)$ be a path in the stability manifold with the property that $\tilde{\pi}(\sigma(t)) \in \overline{K M}(X)$ is a linear degeneration to $[v]$. Let $Y$ be the K3 surface associated to $[v]$ by Ma's theorem [3.10. Then there exist

(1) a derived equivalence $\Phi: \mathcal{D}^{b}(Y) \stackrel{\sim}{\longrightarrow} \mathcal{D}^{b}(X)$

(2) classes $x \in N S(Y)_{\mathbb{R}}, y \in \overline{A m p}(Y)$ and

(3) a path $g(t) \in \widetilde{G l}_{2}^{+}(\mathbb{R})$

such that

for all $t \gg 0$

$$
\sigma(t)=\Phi_{*}\left(\sigma_{Y}^{*}(x, t y) \cdot g(t)\right)
$$

Moreover, the hearts of $\sigma(t) \cdot g(t)^{-1}$ are independent of $t$ for $t \gg 0$. If $y \in \operatorname{Amp}(X)$, then the heart can be explicitly described as the tilt $\mathcal{A}_{Y}(x, y)$ of $\operatorname{Coh}(Y)$.

Proof. By Proposition [5.2, $\tilde{\pi}(\sigma(t))$ is a linear degeneration if and only if

$$
\bar{\pi}(\sigma(t))=\exp _{w}\left(x_{0}+i t y_{0}\right) \in \mathfrak{D}_{0}^{+}(X)
$$

for some $w \in \Gamma_{X} \cdot v, x_{0} \in A(w)_{\mathbb{R}}, y_{0} \in C(L(w))$.

Note that, $w$ is a standard vector and the Hodge structure on $w^{\perp} / w$ induced by $\tilde{H}(X, \mathbb{Z})$ is isomorphic to $v^{\perp} / v$. So, by Theorem 7.11 and Remark 3.11 there is a derived equivalence $\Phi: \mathcal{D}^{b}(X) \rightarrow \mathcal{D}^{b}(Y)$ such that $\Phi^{H}(w)=$ $v_{0}$, and $\Phi_{*} \sigma(t) \in \operatorname{Stab}^{\dagger}(Y)$. Hence we may assume, without loss of generality, that $w=v_{0}$.

Now we claim, that there is a continuous path $g(t) \in \widetilde{G l} l_{2}^{+}(\mathbb{R})$ such that

$$
\pi(\sigma(t) \cdot g(t))=\operatorname{Exp}_{v_{0}}\left(x_{0}+i t y_{0}\right) \in \mathcal{P}_{0}^{+}(X) .
$$

\footnotetext{
3 The stability condition $\sigma_{Y}^{*}(x, y)$ was constructed in Lemma 4.8. If $y \in A m p(X)$, then $\sigma_{Y}^{*}(x, y)$ agrees with Bridgeland's stability condition $\sigma_{Y}(x, y)$ (cf. Definition 4.3)
} 
Moreover, two such paths $g(t), g^{\prime}(t)$ differ by an even shift $\Sigma_{2 k}, k \in \mathbb{Z}$ (cf. Example 4.2), i.e. $g^{\prime}(t)=\Sigma_{2 k} \circ g(t)$.

Indeed, in Remark 2.6 we constructed a section $q_{v_{0}}: \mathfrak{D}(X) \rightarrow \mathcal{P}(X)$ of the $G l_{2}^{+}(\mathbb{R})$-action on $\mathcal{P}(X)$. Hence, there is a unique $h(t) \in G l_{2}^{+}(\mathbb{R})$ such that $\pi(\sigma(t)) \cdot h(t)=q_{v_{0}}\left(\exp _{v_{0}}\left(x_{0}+i t y_{0}\right)\right)=\operatorname{Exp}_{v_{0}}\left(x_{0}+i t y_{0}\right)$. Every choice of a continuous lift $g(t)$ of $h(t)$ to $\widetilde{G l}_{2}^{+}(\mathbb{R})$ has the required property. As $\widetilde{G l}_{2}^{+}(\mathbb{R}) \rightarrow G l_{2}^{+}(\mathbb{R})$ is a Galois cover with Galois group $\mathbb{Z}$ acting by even shifts $k \mapsto \Sigma_{2 k}$ the latter statement follows.

We choose a $t_{0}>0$ such that $\left(t_{0} y_{0}\right)^{2}>2$. Lemma 4.14] shows, that there is an auto-equivalence $\Psi \in \widetilde{W}(X) \subset A u t^{\dagger}\left(\mathcal{D}^{b}(X)\right)$, such that $\Psi_{*} \sigma\left(t_{0}\right) \in \bar{U}(X)$. It is easy to see, that there is a (unique) $k \in \mathbb{Z}$ such that $\Psi_{*} \sigma\left(t_{0}\right) \cdot \Sigma_{2 k} \in$ $\bar{V}(X)$. This allows us to assume, without loss of generality, that $\sigma\left(t_{0}\right) \in$ $\bar{V}(X)$.

By assumption on $t_{0}$, we have furthermore $\sigma\left(t_{0}\right) \in \bar{V}_{>2}(X)$. Now Lemma 4.8 shows that

$$
\sigma\left(t_{0}\right)=\sigma_{X}^{*}\left(x, t_{0} y\right) \text {. }
$$

We claim that the same holds for all $t \geq t_{0}$. Indeed, let $\sigma^{\prime}(t)=\sigma_{X}^{*}(x, t y), t \geq$ $t_{0}$. Then $\sigma(t)$ and $\sigma^{\prime}(t)$ are two lifts of the path $\operatorname{Exp}_{v_{0}}(x+i t y) \in \mathcal{P}_{0}^{+}(X)$ to $\operatorname{Stab}^{\dagger}(X)$ with the same value at $t=t_{0}$. As $\pi$ is a covering-space we have $\sigma(t)=\sigma^{\prime}(t)$. This shows the claim and therefore the first part of the proposition.

It remains to show, that the hearts $\sigma_{X}^{*}(x, t y)$ are independent of $t \geq t_{0}$. In the case $y \in A m p(X)$ this follows directly from Remark 4.4. The general case is more involved:

We introduce the symbol $\mathcal{A}(t)$ for the heart, and $\mathcal{P}_{t}(\phi)$ for the slicing, of the stability stability condition $\sigma(t)$. Let $E \in \mathcal{A}\left(t_{0}\right)$, we have to show, that $E \in \mathcal{A}(t)$ for all $t \geq t_{0}$. We claim the following statements:

(1) If $E \in \mathcal{P}_{t}((0,1))$ for one $t \geq t_{0}$, then $E \in \mathcal{P}_{\tau}([0,1])$ for all $\tau \geq t_{0}$.

(2) If $E \in \mathcal{P}_{t}(0)$ for one $t \geq t_{0}$, then $E \in \mathcal{P}_{\tau}(0)$ for all $\tau \geq t_{0}$.

(3) If $E \in \mathcal{P}_{t}(1)$ for one $t \geq t_{0}$, then $E \in \mathcal{P}_{\tau}(1)$ for all $\tau \geq t_{0}$.

Once we have shown the claim, we argue as follows. Grouping HarderNarasimhan factors in $\sigma(1)$ we get an exact triangle

$$
A \longrightarrow E \longrightarrow B \cdots \cdots \cdots \cdots
$$

with $A \in \mathcal{P}_{t_{0}}(1)$ and $B \in \mathcal{P}_{t_{0}}((0,1))$. Let now $t \geq t_{0}$. By (1) we have $B \in \mathcal{P}_{t}([0,1])$. Taking Hader-Narasimhan filtration in $\sigma(t)$, yields exact triangles

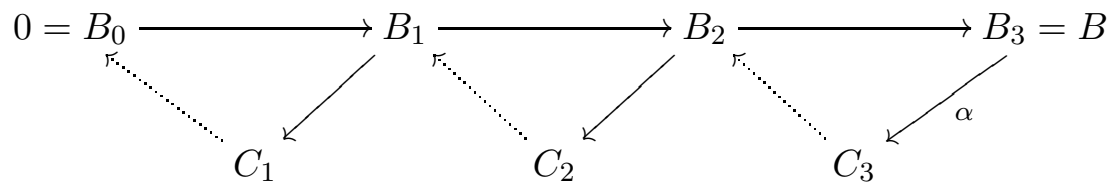

with $C_{1} \in \mathcal{P}_{t}(1), C_{2} \in \mathcal{P}_{t}((0,1)), C_{3} \in \mathcal{P}_{t}(0)$. By (2) we have $C_{3} \in \mathcal{P}_{t_{0}}(0)$, but $B \in \mathcal{P}_{t_{0}}((0,1))$, therefore $\left(\alpha: B \rightarrow C_{3}\right)=0$ which is only possible if $C_{3}=0$. This means that $B \in \mathcal{P}_{t}((0,1])=\mathcal{A}(t)$. By (3) we also have $A \in \mathcal{P}_{t}(1) \subset \mathcal{A}(t)$, which implies $E \in \mathcal{A}(t)$ since $\mathcal{A}(t)$ is extension closed.

\footnotetext{
${ }^{4}$ We use the symbol $A \cdots \cdots \cdots \cdots, B$ for a morphism $A \longrightarrow B[1]$.
} 
Ad 1: Let $\omega \in A m p(X)$ be an ample class, then also $y+s \omega$ is ample for all $s>0$. For $s \geq 0, t \geq t_{0}$, let $\sigma(t, s)=\sigma^{*}(x, t(y+s \omega)) \in \bar{V}(X)$ (cf. Lemma 4.8). Note that $\sigma(t, 0)=\sigma(t)$.

The property $E \in \mathcal{P}_{\sigma}((0,1))$ is clearly open in $\sigma$. Hence we find $\varepsilon>0$, with $E \in \mathcal{P}_{t, s}((0,1))$ for all $\varepsilon>s \geq 0$. If $s>0$, then $\sigma(t, s) \in V(X)$ and the heart $\mathcal{A}(\sigma(t, s))$ is independent of $t \geq t_{0}$ (cf. Remark 4.4). Therefore, $E \in \mathcal{A}(\sigma(\tau, s))$ for all $\tau \geq t_{0}, s>0$. Taking the limit $s \rightarrow 0$ we find $E \in \mathcal{P}_{\tau}([0,1])$, for all $\tau \geq t_{0}$.

Ad 3: Applying a shift we reduce this statement to (2).

Ad 2: The property $E \in \mathcal{P}_{\tau}(0)$ is clearly closed in $\tau$. It suffices to show openness. Fix $t \geq t_{0}$ with $E \in \mathcal{P}_{t}(0)$ and $1>\varepsilon>0$.

Let $T$ be the set of objects which occur as semi-stable factors of $E$ in a stability condition $\sigma(\tau),|t-\tau| \leq \varepsilon$. Then the set $T$ has bounded mass (cf. proof of [Bri08, Prop. 9.3.]), and therefore the set of Mukai vectors $S=\{v(A) \mid A \in T\}$ is finite (cf. [Bri08, Lem. 9.3]).

Writing out formula for $Z_{t}(v)$ as in [Bri08, Sec. 6], we see that $\operatorname{Im}\left(Z_{t}(v)\right)$ vanishes if and only if $\operatorname{Im}\left(Z_{\tau}(v)\right)$ vanishes for all $\tau \geq t_{0}$. It follows that $S$ decomposes as a disjoint union $S=S^{0} \bullet S^{\prime}$, where $\operatorname{Im}\left(Z_{\tau}(v)\right)=0($ or $\neq 0)$ for all $\tau \geq t_{0}$, if $v \in S^{0}$ (or $v \in S^{\prime}$ respectively).

As the interval $[t-\varepsilon, t+\varepsilon]$ is compact and $S$ is finite, there exists a $1>\alpha>0$ such that $\left|\arg \left(Z_{\tau}(v)\right)\right|>\alpha$ for all $v \in S^{\prime},|\tau-t| \leq \varepsilon$.

Making $\varepsilon$ again smaller, we can assume that

$$
E \in \mathcal{P}_{\tau}((-\alpha, \alpha)) \text { for all }|t-\tau| \leq \varepsilon .
$$

It follows that all semi-stable factors $A$ of $E$ in stability condition $\sigma(\tau)$ with $|\tau-t| \leq \varepsilon$, have the property that $v(A) \in S^{0}$. Moreover, as $A \in \mathcal{P}_{\tau}((-\alpha, \alpha))$ and $\arg \left(Z_{\tau}(A)\right) \in \mathbb{Z}$, we find $A \in \mathcal{P}_{\tau}(0)$ and therefore $E \in \mathcal{P}_{\tau}(0)$.

\subsection{Limiting hearts.}

Definition 5.4. Let $\mathcal{C}$ be a category. For a sequence of full subcategories $\mathcal{A}(t) \subset \mathcal{C}, t \gg 0$ define the limit to be the full subcategory of $\mathcal{C}$ with objects

$$
\lim _{t \rightarrow \infty} \mathcal{A}(t)=\{E \in \mathcal{C} \mid E \in \mathcal{A}(t) \text { for all } t \gg 0\} .
$$

Theorem 5.5. Let $[v] \in \overline{K M}(X)$ be a standard cusp, and $Y$ the associated K3 surface. Then, there exist a path $\sigma(t) \in \operatorname{Stab}^{\dagger}(X), t \gg 0$ and an equivalence $\Phi: \mathcal{D}^{b}(Y) \stackrel{\sim}{\longrightarrow} \mathcal{D}^{b}(X)$ such that

(1) $\lim _{t \rightarrow \infty} \tilde{\pi}(\sigma(t))=[v] \in \overline{K M}(X)$ and

(2) $\lim _{t \rightarrow \infty} \mathcal{A}(\sigma(t))=\Phi(\operatorname{Coh}(Y))$

as subcategories of $\mathcal{D}^{b}(X)$.

Proof. By Theorem 7.11 there is a derived equivalence $\Phi: \mathcal{D}^{b}(X) \rightarrow \mathcal{D}^{b}(Y)$ mapping $[v]$ to $\left[v_{0}\right]$ and $\sigma(t)$ into the distinguished component $\operatorname{Stab}^{\dagger}(Y)$. Hence we may assume, without loss of generality, that $[v]=\left[v_{0}\right]$ and $X=Y$.

Let $\omega \in A m p(X)$ and consider the sequence $\sigma_{X}(\omega t, \omega t) \in \operatorname{Stab}^{\dagger}(X)$.

As in the proof of Proposition [5.2, we see that $\tilde{\pi}(\sigma(t))=[\exp (t \beta+i t \omega)]$ converges to $\left[v_{0}\right]$. Indeed, if the vector $\beta+i t \omega$ lies in a principal open $U\left(K, v_{0}\right)$, then also $t \beta+i t \omega \in U\left(K, v_{0}\right)$, since $U(K, v)$ is invariant under the additive action of $L\left(v_{0}\right)_{\mathbb{R}}$ on $T\left(N, v_{0}\right)$. 
The heart $\mathcal{A}_{X}(t \omega, t \omega)$ consists of objects $E \in \mathcal{D}^{b}(X)$ with $H^{0}(E) \in \mathcal{T}(t)$, $H^{-1}(E) \in \mathcal{F}(t)$ and $H^{i}(E)=0$ for all $i \notin\{0,-1\}$, where

$$
\begin{aligned}
& \mathcal{T}(t)=\left\{A \in \operatorname{Coh}(X) \mid A \text { torsion or } \mu_{\omega}^{\min }\left(A / A_{\text {tors }}\right)>t \omega^{2}\right\} \\
& \mathcal{F}(t)=\left\{A \in \operatorname{Coh}(X) \mid A \text { torsion free and } \mu_{\omega}^{\max }(A) \leq t \omega^{2}\right\} .
\end{aligned}
$$

As $\omega^{2}>0$ every sheaf $A$ lies in $\mathcal{F}(t)$ for $t$ sufficiently large. Similarly no sheaf $A$ lies in $\mathcal{T}(t)$ for all $t \gg 0$. Thus we find $\lim _{t \rightarrow \infty} \mathcal{A}_{X}(\omega t, \omega t) \cong C o h(X)[1]$.

5.4. Metric aspects. In this section we will define a natural Riemannian metric on the period domain $\mathfrak{D}(N)$ and show that linear degenerations are geodesics.

Let $N$ be a lattice of signature $(2, \rho)$. The natural action of the real Lie group $G=O\left(N_{\mathbb{R}}\right)$ on $\mathbb{P}\left(N_{\mathbb{C}}\right)$ induces a transitive action of $G$ on $\mathfrak{D}(N)$. Let $[z] \in \mathfrak{D}(N)$ be a point and let $P \subset N_{\mathbb{R}}$ be the positive definite subspace spanned by $\operatorname{Re}(z)$ and $\operatorname{Im}(z)$. The stabilizer of $[z] \in \mathfrak{D}(N)$ is the compact subgroup

$$
K_{P}=\{g \in G \mid g \cdot[z]=[z]\} \cong S O(P) \times O\left(P^{\perp}\right) .
$$

Let $\mathfrak{k}_{P} \subset \mathfrak{g}$ be the Lie algebra of $K_{P} \subset G$. We can identify the tangent space $T_{[z]} \mathfrak{D}(N)$ with the quotient $\mathfrak{g} / \mathfrak{k}_{P}$.

As $G$ is semi-simple, the Killing form $B$ on $\mathfrak{g}$ is non-degenerate. Let $\mathfrak{m}_{P}=\mathfrak{k}_{P}^{\perp}$ be the orthogonal complement of $\mathfrak{k}_{P}$ with respect to $B$. More explicitly, by [Hel78, III.B.ii] we have $B(X, Y)=\rho \cdot \operatorname{Tr}(X \circ Y)$ and

$$
\mathfrak{m}_{P}=\left\{X \in \mathfrak{g} \mid X(P) \subset P^{\perp}, X\left(P^{\perp}\right) \subset P\right\} .
$$

We get a Cartan decomposition $\mathfrak{g}=\mathfrak{k}_{P} \oplus \mathfrak{m}_{P}$. The restriction of $B$ to $\mathfrak{m}_{P}$ is positive definite and induces an invariant Riemannian metric on $\mathfrak{D}(N)$ via the canonical isomorphism $\mathfrak{m}_{P} \cong T_{[z]} \mathfrak{D}(N)$ (cf. Hel78, III.7.7.4]).

Now [Hel78, IV.3, Thm. 3.3.iii] shows, that the geodesics of $\mathfrak{D}(N)$ through $[z]$ are given by the images $\exp (t X) \cdot[z]$ of the one-parameter sub-groups $\{\exp (t X) \mid t \in \mathbb{R}\} \subset G$ with $X \in \mathfrak{m}_{P}$. We will construct a special $X \in \mathfrak{m}_{P}$ such that $\exp (t X) \cdot[z]$ is a linear degeneration through $[z]$.

Let $v_{0} \in N$ be a standard vector and let $x+i y \in T\left(N, v_{0}\right)$ with $[z]=$ $\exp _{v_{0}}(x+i y)$. Recall that $x \in N_{\mathbb{R}} / v_{0} \mathbb{R}$ with $x \cdot v_{0}=-1$. There is a unique lift $x_{0}$ of $x$ to $N_{\mathbb{R}}$ such that $\left(x_{0}\right)^{2}=0$. Indeed, if $\tilde{x}$ is any lift, then $x_{0}=$ $\tilde{x}-\frac{1}{2}\left(\tilde{x}^{2}\right) v_{0}$ has the required property.

Set $x_{1}=-x_{0}$ and let $U \subset N_{\mathbb{R}}$ be the hyperbolic plane spanned by $\left(v_{0}, x_{1}\right)$. We get a one-dimensional Lie sub-algebra

$$
\mathfrak{a}\left(v_{0}, x\right)=\mathfrak{s o}(U) \subset \mathfrak{g}
$$

which depends on the choice of $v_{0}$ and $x$.

Lemma 5.6. The Lie algebra $\mathfrak{a}\left(v_{0}, x\right)$ is contained in $\mathfrak{m}_{P}$.

Proof. Let $R=U^{\perp}$ and decompose $N_{\mathbb{R}}$ as a direct sum $N_{\mathbb{R}}=\left\langle v_{0}\right\rangle \oplus\left\langle x_{1}\right\rangle \oplus R$. We write elements of $N_{\mathbb{R}}$ as column vectors $(a, b, c)^{t r}=a v_{0}+b v_{1}+c$ with $a, b \in \mathbb{R}$ and $c \in R$. We have

$$
\operatorname{Exp}_{v_{0}}(x+i y)=\left(-\frac{1}{2} y^{2},-1, i y\right)^{t r} .
$$


The two-plane $P$ is spanned by the vectors $\left(-\frac{1}{2} y^{2},-1,0\right)^{t r}$ and $(0,0, y)^{t r}$. The Lie algebra $\mathfrak{a}\left(v_{0}, a\right)$ consists of all matrices

$$
A_{\lambda}=\left(\begin{array}{ccc}
\lambda & 0 & 0 \\
0 & -\lambda & 0 \\
0 & 0 & 0
\end{array}\right), \quad \lambda \in \mathbb{R} .
$$

One checks easily, that $A_{\lambda}(P) \perp P$. The orthogonal complement of $P$ consists of all vectors $\gamma=\left(\frac{1}{2} b y^{2}, b, c\right)^{t r}$ with $c . y=0$. Therefore,

$$
A_{\lambda}(\gamma)=\left(\lambda \frac{1}{2} b y^{2},-\lambda b, 0\right)^{t r}=\lambda b \alpha \in P .
$$

This shows that $\mathfrak{a}\left(v_{0}, x\right) \subset \mathfrak{m}_{P}$.

Lemma 5.7. Let $[z]=\exp _{v_{0}}(x+i y) \in \mathfrak{D}(N)$ and $A_{\lambda} \in \mathfrak{a}\left(v_{0}, x\right)$, then the action of $\exp \left(A_{\lambda}\right)$ is given by

$$
\exp \left(A_{\lambda}\right) \cdot[z]=\exp _{v_{0}}(x+i t y)
$$

where $t=\exp (\lambda)$.

Proof. As above we write elements of $N_{\mathbb{R}}$ as column vectors with respect to the decomposition $N_{\mathbb{R}}=\left\langle v_{0}\right\rangle \oplus\left\langle x_{1}\right\rangle \oplus R$. Similarly, endomorphisms are represented by matrices. We have

$$
\exp \left(A_{\lambda}\right)=\left(\begin{array}{ccc}
t & 0 & 0 \\
0 & t^{-1} & 0 \\
0 & 0 & i d_{R}
\end{array}\right),
$$

where $t=\exp (\lambda)$. Therefore,

$$
\begin{aligned}
\exp \left(A_{\lambda}\right) \cdot P & =\left\langle\left(-t \frac{1}{2} y^{2},-t^{-1}, 0\right)^{t r},(0,0, y)^{t r}\right\rangle \\
& =\left\langle\left(-\frac{1}{2}(t y)^{2},-1,0\right)^{t r},(0,0, t y)^{t r}\right\rangle
\end{aligned}
$$

which is the two-plane spanned by the real- and imaginary parts of the vector $\operatorname{Exp}_{v_{0}}(x+i t y)$.

Corollary 5.8. For all $x+i y \in T\left(N, v_{0}\right)$, the path

$$
\alpha(t)=\exp _{v_{0}}(x+i \exp (t) y) \in \mathfrak{D}(N)
$$

is a geodesic of constant speed.

If $\Gamma \subset G$ is a discrete subgroup acting properly and discontinuously on $\mathfrak{D}(N)$ then the quotient $\Gamma \backslash \mathfrak{D}(N)$ inherits a Riemannian metric on the smooth part $(\Gamma \backslash \mathfrak{D}(N))_{\text {reg }}$. Geodesics in $(\Gamma \backslash \mathfrak{D}(N))_{\text {reg }}$ are locally the images of geodesics on $\mathfrak{D}(N)$. More generally we define geodesics in $\Gamma \backslash \mathfrak{D}(N)$ to be the images of geodesics in $\mathfrak{D}(N)$.

This discussion applies in particular to the Kähler moduli space of a K3 surface $X$. From the definition of linear degeneration and Corollary 5.8 we get immediately the following statement.

Corollary 5.9. Linear degenerations are geodesics in the Kähler moduli space $K M(X)$. 
Note however, that our parametrization $\exp (x+i t y)$ is not of constant speed.

We conjecture the following converse to the above corollary.

Conjecture 5.10. Let $[v] \in \overline{K M}(X)$ be a zero-dimensional cusp. Then every geodesic converging to $[v]$ is a linear degeneration.

We have the following evidence. The conjecture holds true in the case $X$ has Picard rank one. Then, $\mathfrak{D}(N)^{+}$is isomorphic to the upper half plane and the geodesics converging to the cusp $i \infty$ are precisely the vertical lines, which are our linear degenerations.

If one uses the reductive Borel-Serre compactification $\overline{K M}(X)^{B S}$ to compactify $K M(X)$, then the analogues conjecture seems to follow from JM02. Indeed, Ji and MacPherson describe the boundary of $\overline{K M}(X)^{B S}$ as a set of equivalence classes of, so called, EDM-geodesics (cf. JJM2, Prop. 14.16]). Moreover, all EDM-geodesics are classified in [JM02, Thm. 10.18]. They are of the form $(u, z, \exp (t H)) \in N_{Q} \times X_{Q} \times A_{Q}$ where $Q \subset G$ is a rational parabolic subgroup and $N_{Q} \times X_{Q} \times A_{Q} \cong \mathfrak{D}(N)$ is the associated horocycle decomposition. We think, that linear degenerations to $[v]$ are the geodesics associated to the stabilizer group $G_{[v]}$ of $[v] \in \mathbb{P}\left(N_{\mathbb{C}}\right)$. Moreover, all geodesics $\gamma$ that converge to the boundary component $e([v]) \subset \overline{K M}(X)^{B S}$ associated to $G_{[v]}$ should have the EDM property. It follows form the classification, that $\gamma$ is of the form $(u, z, \exp (t H))$ for some rational parabolic subgroup $Q \subset G$. Since $\gamma$ converges to $e([v])$, we have $Q=G_{[v]}$ and therefore $\gamma$ should be a linear degeneration.

There is a natural map $\overline{K M}(X)^{B S} \rightarrow \overline{K M}(X)$ (cf. [BJ06, III.15.4.2]). One should be able to prove the full conjecture by studying the fibers of this map over a cusp $[v] \in \overline{K M}(X)$.

\section{Moduli spaces of COMplexes on K3 Surfaces}

In this section we construct K3 surfaces as moduli spaces of stable objects in the derived category of another K3 surface. First we introduce a moduli functor, which is a set-valued version of Lieblich's moduli stack cf. [Lie06]. We will show in subsection 6.3, that Fourier-Mukai equivalences induce natural isomorphisms between moduli spaces. Finally, in subsection 6.5 we prove our main theorem.

Before we can give the actual definition, we recall the notion of a perfect complex in the first subsection. Moreover, we establish a base-change formula and a semi-continuity result which will be important later.

6.1. Perfect complexes. We denote by $\mathcal{D}(X)$ the unbounded derived category of coherent sheaves on $X$.

Definition 6.1. Let $X \rightarrow T$ be a morphism of schemes. A complex $E \in$ $\mathcal{D}(X)$ is called relatively $T$-perfect, if there is an open cover $\left\{U_{\nu}\right\}$ of $X$ such that $\left.E\right|_{U_{\nu}}$ is quasi-isomorphic to a bounded complex of $T$-flat sheaves of finite presentation.

We call $E$ strictly $T$-perfect if $E$ itself is quasi-isomorphic to a bounded complex of $T$-flat sheaves of finite presentation. 
Lemma 6.2. [Lie06, Cor. 2.1.7] If $T$ is an affine scheme and $f: X \rightarrow T$ is a flat, finitely presented and quasi-projective morphism, then every relatively $T$-perfect complex is strictly T-perfect.

The following base-change result is presumably well known to the experts. The main difference to the usual base change theorems like Har77, Prop. 5.2 ] is that we do not assume flatness of any maps, but perfectness of the complex.

Proposition 6.3 (Base Change). Consider a diagram of separated, noetherian schemes

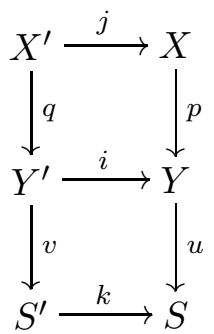

where $p$ is proper and both squares are Cartesian. Let $E \in \mathcal{D}^{b}(X)$ be a strictly $S$-perfect complex. Then the base change morphism

$$
\mathbb{L} i^{*} \mathbb{R} p_{*} E \longrightarrow \mathbb{R} q_{*} \mathbb{L} j^{*} E
$$

is an isomorphism.

The same holds true if $E \in \mathcal{D}^{b}(X)$ is only $S$-perfect but $p$ is flat, finitely presented and projective.

Proof. As the statement is local in $Y^{\prime}$ we may assume that $Y, Y^{\prime}, S, S^{\prime}$ are affine. If $E$ is $S$-perfect and $p$ is flat, finitely-presented and projective, then Lemma 6.2 shows that $E$ is strictly $S$-perfect. Hence it suffices to treat the case that $E$ is a bounded complex of $S$-flat coherent sheaves on $X$.

Step 0) Choose a finite open affine cover $\mathfrak{U}=\left\{U_{\nu}\right\}$ of $X$. The Cechcomplex $\mathcal{C}(\mathfrak{U}, E) \in \mathcal{D}^{b}(X)$ of $E$ with respect to $\mathfrak{U}$ is the total complex of the following double complex of quasi-coherent sheaves on $X$

$$
\mathcal{C}^{q}\left(\mathfrak{U}, E^{p}\right)=\left.\prod_{\nu_{0}<\cdots<\nu_{q}} \iota_{*} E^{p}\right|_{U_{\nu_{0}} \cap \cdots \cap U_{\nu_{q}}}, \quad d_{1}^{p q}=d_{E}^{p}, d_{2}^{p q}=(-1)^{p} \delta^{q},
$$

where $\iota: U_{\nu_{0}} \cap \cdots \cap U_{\nu_{q}} \rightarrow X$ denotes the inclusion. It comes with a canonical morphism

$$
E \longrightarrow \mathcal{C}(\mathfrak{U}, E), \quad m \in E^{p} \mapsto\left(\left.m\right|_{U_{\nu}}\right)_{\nu} \in \mathcal{C}^{0}\left(\mathfrak{U}, E^{p}\right) .
$$

which is a quasi-isomorphism. This can be checked using the spectral sequence for double complexes and the vanishing of the $E_{2}^{p q}$ in degrees $q \neq 0$.

Step 1) We claim that the sheaves $\mathcal{C}^{n}(\mathfrak{U}, E), n \in \mathbb{Z}$ are acyclic for $p_{*}$. The sheaf $\mathcal{C}^{n}(\mathfrak{U}, E)$ is a direct sum of sheaves of the form $\left.\iota_{*} E^{p}\right|_{U^{\prime}}$ where $U^{\prime}=U_{\nu_{0}} \cap \cdots \cap U_{\nu_{n}}$. Since $X$ is separated $U^{\prime}$ is affine. The morphism $p^{\prime}: U^{\prime} \longrightarrow Y$ between affine schemes is affine and hence all higher direct images $\left.\mathbb{R}^{i} p^{\prime} E^{p}\right|_{U^{\prime}}=0, i>0$ vanish. Hence

$$
\mathbb{R} p_{*} E \cong p_{*} \mathcal{C}(\mathfrak{U}, E) .
$$


The sheaves $\mathcal{C}^{n}(\mathfrak{U}, E)$ are still $S$-flat, since $\left.p_{*} E\right|_{U^{\prime}}$ are given by restriction of scalars along the morphism of affine schemes $p^{\prime}: U^{\prime} \rightarrow Y$. This shows that

$$
\mathbb{L} i^{*} \mathbb{R} p_{*} E \cong i^{*} p_{*} \mathcal{C}(\mathfrak{U}, E) .
$$

Step 2) On the other hand we have

$$
\mathbb{L} j^{*} E \cong j^{*} \mathcal{C}(\mathfrak{U}, E),
$$

since $\mathcal{C}^{n}(\mathfrak{U}, E)$ is $S$-flat for all $n \in \mathbb{Z}$.

We claim that $j^{*} \mathcal{C}^{n}(\mathfrak{U}, E), n \in \mathbb{Z}$ are acyclic for $q_{*}$.

Again we use that $j^{*} \mathcal{C}^{n}(\mathfrak{U}, E)$ are direct sums of sheaves of the form $\left.j^{*} \iota_{*} E^{p}\right|_{U^{\prime}}$ with $U^{\prime}=U_{\nu_{0}} \cap \cdots \cap U_{\nu_{q}}$. Consider the open affine subset $V^{\prime}=$ $j^{-1}\left(U^{\prime}\right) \subset X^{\prime}$ and the following diagram

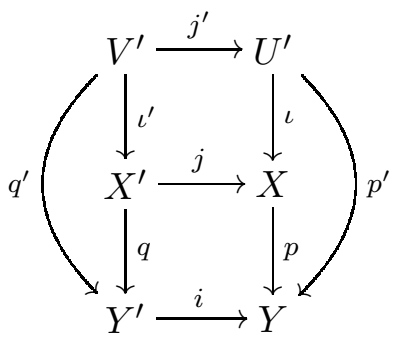

We have

$$
\left.j^{*} \iota_{*} E^{p}\right|_{U^{\prime}}=j^{*} \iota_{*} \iota^{*} E^{p}=\iota_{*}^{\prime} j^{\prime *} \iota^{*} E^{p}=\iota_{*}^{\prime} \iota^{*} j^{*} E^{p} .
$$

In the second step we use base change for open inclusions of affine schemes into separated schemes. It follows that the higher direct images vanish:

$$
\mathbb{R}^{i} q_{*}\left(\left.j^{*} \iota_{*} E^{p}\right|_{U^{\prime}}\right)=\mathbb{R}^{i} q_{*}\left(\iota_{*}^{\prime} \iota^{\prime *} j^{*} E^{p}\right)=\mathbb{R}^{i} q_{*}^{\prime}\left(\iota^{\prime *} j^{*} E^{p}\right)=0
$$

for all $i>0$. We used in the second step that $\iota_{*}$ is exact and in the third step that $q^{\prime}$ is affine.

This shows that

$$
\mathbb{R} q_{*} \mathbb{L} j^{*} E \cong q_{*} j^{*} \mathcal{C}(\mathfrak{U}, E) .
$$

Step 3) The base change morphism

$$
\theta: \mathbb{L} i^{*} \mathbb{R} p_{*} E \longrightarrow \mathbb{R} q_{*} \mathbb{L} j^{*} E
$$

can be constructed using the adjunction of $\mathbb{L} q^{*}, \mathbb{R} q_{*}$, and $\mathbb{L} p^{*}, \mathbb{R} p_{*}$. It can be computed on appropriate resolutions using the adjunction of functors of sheaves between $q^{*}, q_{*}$ and $p^{*}, p_{*}$.

Under the quasi-isomorphisms (44) and (3) the morphism $\theta$ is given by a morphism of complexes whose components are base-change morphisms

$$
\left.q_{*} j^{*} \iota_{*} E^{p}\right|_{U^{\prime}}=\left.\left.q_{*}^{\prime} j^{\prime *} E^{p}\right|_{U^{\prime}} \longrightarrow i^{*} p_{*}^{\prime} E^{p}\right|_{U^{\prime}}=\left.i^{*} p_{*} \iota_{*} E^{p}\right|_{U^{\prime}}
$$

for the affine schemes $V^{\prime}, U^{\prime}, Y, Y^{\prime}$ and thus isomorphisms.

Proposition 6.4 (Semi-continuity). Let $X \rightarrow T$ be a proper morphism between separated, noetherian schemes and let $E \in \mathcal{D}^{b}(X)$ be a T-perfect complex.

For $t \in T$, denote by $i_{t}: X_{t}=X \times_{T}\{t\} \rightarrow X$ the inclusion of the fiber and by $E_{t}=\mathbb{L} i_{t}^{*} E$ the derived restriction. Then the function

$$
\phi^{i}: T \rightarrow \mathbb{Z}: t \mapsto \operatorname{dim}_{k(t)} \mathbb{H}^{i}\left(X_{t}, E_{t}\right)
$$


is upper semi-continuous.

Proof. We may assume $T$ is affine and $E$ is a bounded complex of $T$-flat coherent sheaves. By Theorem 6.3 we have

$$
\mathbb{H}^{i}\left(X, E_{t}\right)=\mathcal{H}^{i}\left(\mathbb{L} i_{t}^{*} \mathbb{R} p_{*} E\right)
$$

as sheaves on $\{t\}=\operatorname{Spec}(k(t))$. In the proof of this theorem we saw that $\mathbb{R} p_{*} E$ can be represented by a bounded complex of $T$-flat, quasi-coherent sheaves with coherent cohomology.

The remaining arguments are identical to the proof of the semi-continuity theorem for a $T$-flat sheaf $E$ in [Har77, Thm. III.12.8].

6.2. Moduli functor. Let $X$ be a K3 surface and $T$ be a scheme over $\mathbb{C}$. For a point $t \in T(\mathbb{C})$ we denote by $i_{t}: X \rightarrow X \times T$ the inclusion of the fiber and for a complex $E \in \mathcal{D}^{b}(X \times T)$ let $E_{t}=\mathbb{L} i_{t}^{*} E$ be the restriction.

Definition 6.5. For $v \in N(X)$ and $\sigma \in \operatorname{Stab}(X)$ consider the moduli functor

$$
\mathcal{M}_{X}^{\sigma}(v):(S h m / \mathbb{C})^{o p} \rightarrow(S e t), \quad T \mapsto\left\{E \in \mathcal{D}^{b}(X \times T) \mid(*)\right\} / \sim .
$$

Here $(S h m / \mathbb{C})$ is the category of separated schemes of finite type over $\mathbb{C}$ The symbol $(*)$ stands for the following conditions.

(1) The complex $E$ is relatively $T$-perfect.

(2) For all $t \in T(\mathbb{C})$ the restriction $E_{t} \in \mathcal{D}^{b}(X)$ is $\sigma$-stable of Mukai vector $v\left(E_{t}\right)=v$.

The equivalence relation $\sim$ is defined as follows. We have $E \sim E^{\prime}$ if and only if there is an open cover $\cup T_{\nu}=T$ of $T$ such that for all $\nu$ there is a line bundle $L \in \operatorname{Pic}\left(T_{\nu}\right)$ and an even number $k \in 2 \mathbb{Z}$ with $E \cong E^{\prime}[k] \otimes p r_{2}^{*} L$ on $X \times T_{\nu}$.

To a morphism of schemes $i: S \rightarrow T$ in $(S h m / \mathbb{C})$ the functor assigns the map

$$
\mathbb{L} i_{X}^{*}: \mathcal{M}_{X}^{\sigma}(v)(T) \longrightarrow \mathcal{M}_{X}^{\sigma}(v)(S)
$$

sending $E \in \mathcal{D}^{b}(X \times T)$ to $\mathbb{L} i_{X}^{*} E$, where $i_{X}=\operatorname{id}_{X} \times i$.

\subsection{Moduli spaces under Fourier-Mukai transformations.}

Theorem 6.6. Let $\Phi: \mathcal{D}^{b}(X) \rightarrow \mathcal{D}^{b}(Y)$ be a Fourier-Mukai equivalence between two $K 3$ surfaces $X$ and $Y$, then $\Phi$ induces an isomorphism of functors

$$
\mathcal{M}_{X}^{\sigma}(v) \stackrel{\cong}{\longrightarrow} \mathcal{M}_{Y}^{\Phi_{*} \sigma}\left(\Phi_{*}^{H} v\right)
$$

Proof. Denote by $p_{T}, q_{T}$ and $\pi$ the projections from $X \times Y \times T$ to $X \times T, Y \times T$ and $X \times Y$, respectively. Let $\mathcal{P} \in \mathcal{D}^{b}(X \times Y)$ be the Fourier-Mukai kernel of $\Phi$. We claim that the map

$$
\mathcal{M}_{X}^{\sigma}(v)(T) \ni E \mapsto \Phi_{T}(E):=\mathbb{R} q_{T *}\left(p_{T}^{*} E \otimes^{\mathbb{L}} \pi^{*} \mathcal{P}\right)
$$

induces a natural transformation $\mathcal{M}_{X}^{\sigma}(v) \rightarrow \mathcal{M}_{Y}^{\Phi_{*} \sigma}\left(\Phi_{*}^{H} v\right)$ between the moduli functors. For this we need to check the following properties.

(1) The complex $\Phi_{T}(E)$ is relatively $T$-perfect.

\footnotetext{
${ }^{5}$ For technical reasons related to Proposition 6.3 we have to restrict ourselves to this subcategory.
} 
(2) Naturality: For all $i: S \rightarrow T \in(S h m / \mathbb{C})$ and $E \in \mathcal{M}_{X}^{\sigma}(v)(T)$ we have

$$
\mathbb{L} i_{Y}^{*} \circ \Phi_{T}(E)=\Phi_{S} \circ \mathbb{L} i_{X}^{*}(E) .
$$

(3) For all $t \in T(\mathbb{C})$ the complex $\Phi_{T}(E)_{t}$ is $\Phi_{*} \sigma$-stable of Mukai vector $\Phi^{H}(v)$.

Ad 1) As $X \times Y$ is a smooth projective scheme, we can represent $\mathcal{P}$ by a bounded complex of coherent, locally free sheaves. Since locally-free sheaves are acyclic for $\_\otimes^{\mathbb{L}}$ - we find that $\pi^{*} \mathcal{P} \otimes^{\mathbb{L}} p_{T}^{*} E$ is $T$-perfect.

Now, Gro71, SGA 6,III,4.8] shows that the pushforward $\mathbb{R} q_{T *}\left(p_{T}^{*} E \otimes^{\mathbb{L}}\right.$ $\left.\pi^{*} \mathcal{P}\right)$ is still $T$-perfect.

Ad 2) This is a direct computation using the base-change formula Proposition 6.3.

Ad 3) By (2) we have $\Phi_{T}(E)_{t} \cong \Phi\left(E_{t}\right)$. Now (3) follows from the definition of $\Phi_{*} \sigma$ and $\Phi^{H}(v)$.

Finally we need to show that (5) is an isomorphism. For this we use the following straight forward Lemma.

Lemma 6.7. Let $\Phi: \mathcal{D}^{b}(X) \rightarrow \mathcal{D}^{b}(Y)$ and $\Psi: \mathcal{D}^{b}(Y) \rightarrow \mathcal{D}^{b}(Z)$ be derived equivalences between $K 3$ surfaces $X, Y, Z$, then

$$
\Phi_{T} \circ \Psi_{T}=(\Phi \circ \Psi)_{T}: \mathcal{M}_{X}^{\sigma}(v) \longrightarrow \mathcal{M}_{Z}^{\tau}(w)
$$

where $w=\Psi^{H}\left(\Phi^{H}(v)\right)$ and $\tau=\Psi_{*}\left(\Phi_{*}(\sigma)\right)$.

An inverse to the equivalence $\Phi$ is given by a Fourier-Mukai transformation $\Psi$ with kernel $\mathcal{P}^{\vee}[2]$. Moreover, the kernels of the compositions $\Psi \circ \Phi$, $\Phi \circ \Psi$ are quasi-isomorphic to $\mathcal{O}_{\Delta} \in \mathcal{D}^{b}(X \times X)$ and $\mathcal{O}_{\Delta} \in \mathcal{D}^{b}(Y \times Y)$ respectively (cf. Huy06, 5.7, ff.]). Clearly $\mathcal{O}_{\Delta}$ induces the identity on $\mathcal{M}_{X}^{\sigma}(v), \mathcal{M}_{Y}^{\sigma}(v)$. This shows that $\Phi_{T}$ and $\Psi_{T}$ are inverse natural transformations.

6.4. More on stability conditions. Before we can finally state our main result on moduli spaces of stable objects we need another digression on stability conditions. First, we prove a classification result for semi-stable objects, then we introduce $v_{0}$-general stability conditions and derive some basic properties.

Proposition 6.8. Let $\sigma \in U(X)$ be a stability condition. Then an object $E$ is $\sigma$-semi-stable with Mukai vector $v_{0}=(0,0,1)$ if and only if there is an $x \in X$ and $k \in 2 \mathbb{Z}$ such that $E \cong \mathcal{O}_{x}[k]$.

$$
\left\{E \in \mathcal{D}^{b}(X) \mid v(E)=v_{0}, E \sigma \text {-semi-stable }\right\}=\left\{\mathcal{O}_{x}[2 k] \mid x \in X, k \in \mathbb{Z}\right\} .
$$

Proof. Let $\sigma \in U(X)$ be a stability condition. The objects $\mathcal{O}_{x}, x \in X$ are $\sigma$-stable by Theorem 4.6 and hence in particular semi-stable.

Let $E^{\prime}$ be a $\sigma$-semi-stable object with Mukai vector $v_{0}$. Applying an element in $\widetilde{G l}_{2}^{+}(\mathbb{R})$ we can assume that $\sigma$ is of the form $\sigma(\omega, \beta)$. There is a unique $k \in \mathbb{Z}$ such that $E^{\prime}[k]=E$ lies in the heart $\mathcal{A}(\omega, \beta)$. As $Z_{\sigma}(E)=$

\footnotetext{
${ }^{6}$ Grothendieck and Illusie use a slightly different definition of relative perfectness. The definition agrees with ours in the case of flat morphisms of finite type between locally noetherian schemes (cf. Lie06, Def. 2.1.1.] ff). The projections $X \times T \rightarrow T$ and $Y \times T \rightarrow T$ clearly have this property.
} 
$(-1)^{k} Z_{\sigma}\left(v_{0}\right)=-(-1)^{k}$ has to lie in $\mathbb{H} \cup \mathbb{R}_{<0}$ the number $k$ has to be even and the phase of $E$ is one. Take a Jordan-Hölder filtration

$$
0 \subset E_{1} \subset E_{2} \subset \cdots \subset E_{n}=E
$$

of $E$ in $\mathcal{A}(\omega, \beta)$. The stable quotients $A_{i}=E_{i} / E_{i-1}$ have the same phase as $E$. Hence we can use the classification result of Huybrechts, Huy08, Prop. 2.2], which shows that $A_{i}=F[1]$ for a vector bundle $F$ or $A_{i}=\mathcal{O}_{x}$ for some $x \in X$ Note that the Mukai vectors in these two cases are given by

$$
v(F[1])=-(r, l, s) \text { with } r>0, \quad v\left(\mathcal{O}_{x}\right)=(0,0,1) .
$$

By assumption we have $\sum_{i} v\left(A_{i}\right)=v(E)=(0,0,1)$. Hence the sum over the ranks of all occurring vector bundles has to be zero. This is only possible if there are none of them. Hence $E$ is an extension of skyscraper sheaves. Comparing Mukai vectors again, one sees that $E$ has to be of the form $\mathcal{O}_{x}$ for some $x \in X$.

Definition 6.9. Fix a Mukai vector $v \in N(X)$. A stability condition $\sigma \in$ $\operatorname{Stab}^{\dagger}(X)$ is called $v$-general if every $\sigma$-semi-stable object $E$ of Mukai vector $v(E)=v$ is $\sigma$-stable.

Lemma 6.10. Every stability condition $\sigma \in U(X)$ is $v_{0}=(0,0,1)$-general. No stability condition $\sigma \in \partial U(X) \subset S t a b^{\dagger}(X)$ is $v_{0}$-general.

Proof of Lemma. By Proposition 6.8 all $\sigma$-semi-stable objects of Mukai vector $v_{0}$ are shifts of skyscraper sheaves. All skyscraper sheaves $\mathcal{O}_{x}$ are $\sigma$-stable by Proposition 4.6.

For the second claim note that $\mathcal{O}_{x}$ remains semi-stable for $\sigma \in \bar{U}(X)$. If $\sigma \in \bar{U}(X)$ and all $\mathcal{O}_{x}$ are $\sigma$-stable, then $\sigma \in U(X)$ by Proposition 4.6, Hence for $\sigma \in \partial U(X)$ there are strictly semi-stable skyscraper sheaves. This means $\sigma$ is not $v_{0}$-general.

Lemma 6.11. For all primitive Mukai vectors $v \in N(X)$ the set of $v$-general stability conditions is dense and open in $\operatorname{Stab}^{\dagger}(X)$.

Proof. Choose an open subset $B^{\circ}$ with compact closure $B$. In the proof of [Bri08, Prop. 9.3.] it is shown that

$$
S=\left\{E \in \mathcal{D}^{b}(X) \mid E \sigma \text {-semi-stable for some } \sigma \in B, v(E)=v\right\}
$$

has bounded mass. Hence [Bri08, Prop. 9.3] applies and we get a wall an chamber structure on $B$ such that all objects $E \in S$ are stable outside a locally finite collection of walls. This shows the density. The openness follows from [Bri08, Prop. 9.4] applied to $S$.

\subsection{Reconstruction theorem.}

Theorem 6.12. Let $v \in N(X)$ be a standard vector and $\sigma \in \operatorname{Stab}^{\dagger}(X)$ a $v$-general stability condition.

(1) There exists a K3 surface $Y$ and an isomorphism of functors

$$
\mathcal{M}_{X}^{\sigma}(v) \cong \underline{Y}
$$

where $\underline{Y}$ is the functor $(\operatorname{Shm} / \mathbb{C})^{o p} \rightarrow($ set $): T \mapsto M o r(T, Y)$.

(2) The Hodge structure $H^{2}(Y, \mathbb{Z})$ is isomorphic to the subquotient of $\tilde{H}(X, \mathbb{Z})$ given by $v^{\perp} / v$. 
(3) The universal family $E \in \mathcal{M}_{X}^{\sigma}(v)(Y) \subset \mathcal{D}^{b}(X \times Y)$ induces a derived equivalence $\mathcal{D}^{b}(X) \rightarrow \mathcal{D}^{b}(Y)$.

Proof. The proof consists of three steps. First, we treat the case $v=v_{0}, \sigma \in$ $U(X)$ and show that $\mathcal{M}_{\sigma}\left(v_{0}\right) \cong \underline{X}$ using Proposition 6.8. Next we generalize to $v=v_{0}$ and $\sigma \in \operatorname{Stab}^{\dagger}(X)$ using Lemma 4.14. Finally the general case can be reduced to $v=v_{0}$ using Theorem 7.11

Step 1: Assume that $v_{0}=(0,0,1) \in N(X)$, and $\sigma \in U(X)$, then

$$
\mathcal{M}^{\sigma}\left(v_{0}\right) \cong \underline{X} \text {. }
$$

Indeed, the morphism $\underline{X} \rightarrow \mathcal{M}_{X}^{\sigma}\left(v_{0}\right)$ is given by

$$
f: T \rightarrow X \quad \mapsto \mathcal{O}_{\Gamma_{f}} \in \mathcal{M}_{X}^{\sigma}\left(v_{0}\right)(T)
$$

where $\Gamma_{f} \subset X \times T$ is the graph of $f$. We have to show this map is an isomorphism.

Injectivity: If we have two morphisms $f, g: T \rightarrow X$ with $\mathcal{O}_{\Gamma_{f}} \sim \mathcal{O}_{\Gamma_{g}}$ then we claim that $f=g$. Indeed, by assumption there is a quasi-isomorphism

$$
\mathcal{O}_{\Gamma_{f}} \cong \mathcal{O}_{\Gamma_{g}}[k] \otimes \operatorname{pr}_{2}^{*} L \quad \text { in } \quad \mathcal{D}^{b}(X \times T)
$$

for some $k \in 2 \mathbb{Z}, L \in \operatorname{Pic}(T)$. As $\mathcal{O}_{\Gamma_{f}}$ and $\mathcal{O}_{\Gamma_{g}} \otimes \operatorname{pr}_{2}^{*} L$ are sheaves, we have $k=0$ and the quasi-isomorphism is an isomorphism of coherent sheaves. Moreover,

$$
L=\operatorname{pr}_{2 *}\left(\mathcal{O}_{\Gamma_{g}} \otimes \operatorname{pr}_{2}^{*} L\right) \cong \operatorname{pr}_{2 *}\left(\mathcal{O}_{\Gamma_{f}}\right)=\mathcal{O}_{T}
$$

Hence it is $\mathcal{O}_{\Gamma_{f}} \cong \mathcal{O}_{\Gamma_{g}}$ and it follows that $f=g$.

Surjectivity: If $[E] \in \mathcal{M}_{X}^{\sigma}\left(v_{0}\right)(T)$, then $\mathbb{L} i_{t}^{*} E$ is $\sigma$-stable of Mukai vector $v_{0}$. It follows from Proposition 6.8 that $\mathbb{L} i_{t}^{*} E \cong \mathcal{O}_{x}[k]$ for a point $x \in X$ and $k \in 2 \mathbb{Z}$ depending on $t$. Using the semi-continuity of $t \mapsto h^{i}\left(X, \mathbb{L} i_{t}^{*} E\right)$ (Proposition 6.4) it is easy to see that the shift $k \in 2 \mathbb{Z}$ is independent of $t$ in each connected component $T_{0} \subset T$. Now we argue as in [Huy06, Cor. 5.23.] to see that there is a morphism $f: T_{0} \rightarrow X$ line bundle $L \in P i c\left(T_{0}\right)$ such that $\left.E\right|_{T_{0}} \cong \mathcal{O}_{\Gamma_{f}} \otimes \operatorname{pr}_{2}^{*} L$. Therefore $E \sim \mathcal{O}_{\Gamma_{f}}$ for some $f: T \rightarrow X$.

Step 2. Assume that $\sigma \in \operatorname{Stab}^{\dagger}(X)$ is a $v_{0}$-general stability condition. Then $\mathcal{M}^{\sigma}\left(v_{0}\right)$ is isomorphic to $X$.

Indeed, by Lemma 4.14 we find a $\Phi \in \widetilde{W}(X)$ such that $\sigma^{\prime}=\Phi_{*}(\sigma) \in \bar{U}(X)$. Also note that we have

$$
T_{\mathcal{O}_{C}(k)}^{H}\left(v_{0}\right)=v_{0}, \quad T_{A}^{2 H}\left(v_{0}\right)=v_{0} .
$$

It follows that $v_{0}$-general stability conditions are mapped to $v_{0}$-general stability conditions. By Lemma 6.10 we conclude that $\sigma^{\prime} \in U(X)$ and not in $\partial U(X)$.

As we have seen in Theorem 6.6 the Fourier-Mukai equivalence $\Phi^{-1}$ induces isomorphisms of functors

$$
\left.\mathcal{M}^{\sigma}\left(v_{0}\right) \cong \mathcal{M}^{\Phi_{*} \sigma}\left(\Phi_{*}^{H} v_{0}\right)\right)=\mathcal{M}^{\sigma^{\prime}}\left(v_{0}\right) \cong \underline{X} .
$$

The last isomorphism is provided by step 1 .

Step 3. General case. Let $v$ be a standard vector and $\sigma \in \operatorname{Stab}^{\dagger}(X)$ be a $v$-general stability condition. By Theorem 7.11 there is a $\mathrm{K} 3$ surface $Y$ with Hodge structure $H^{2}(Y, \mathbb{Z}) \cong v^{\perp} / v$ and a derived equivalence $\Phi: \mathcal{D}^{b}(X) \rightarrow$ $\mathcal{D}^{b}(Y)$ respecting the distinguished component and mapping $v \in N(X)$ to $v_{0} \in N(Y)$. 
Since $\sigma \in \operatorname{Stab}^{\dagger}(X)$ is $v$-general also $\Phi_{*}(\sigma) \in \operatorname{Stab}^{\dagger}(Y)$ is $v_{0}$-general. By Theorem 6.6 the Fourier-Mukai transformation $\Phi: \mathcal{D}^{b}(X) \rightarrow \mathcal{D}^{b}(Y)$ induces an isomorphism of moduli functors $\mathcal{M}_{X}^{\sigma}(v) \cong \mathcal{M}_{Y}^{\Phi * \sigma}\left(v_{0}\right)$. Now we apply step 2 to conclude that $\mathcal{M}_{Y}^{\Phi_{*} \sigma}\left(v_{0}\right) \cong \underline{Y}$.

It remains to show that the universal family induces a derived equivalence. This follows from the fact that, under the isomorphism $\underline{Y} \cong \mathcal{M}_{X}^{\sigma}(v)$ the element $i d_{Y} \in \underline{Y}(Y)$ maps to the kernel of the composition of the FourierMukai equivalences used in the various reduction steps.

Remark 6.13. In general one expects that the moduli space $\mathcal{M}^{\sigma}(v)$ undergoes (birational) transformations, called wall-crossings when $\sigma$ moves in $\operatorname{Stab}^{\dagger}(X)$. This behavior can be observed in our situation, too, but the transformations turn out to be isomorphisms.

If $\sigma \in U(X)$, then $\mathcal{M}^{\sigma}\left(v_{0}\right)$ parametrizes the skyscraper sheaves $\mathcal{O}_{x}, x \in$ $X$. When $\sigma$ passes over a wall of type $\left(C_{k}\right)$, then the sheaves $\mathcal{O}_{x}, x \in C$ are replaced by the complexes $T_{\mathcal{O}_{C}(k)} \mathcal{O}_{x}$, whereas the sheaves $\mathcal{O}_{x}, x \notin C$ remain stable.

If $\sigma$ moves over an $(A)^{ \pm}$-type wall, then all sheaves $\mathcal{O}_{x}$ are replaced by the spherical twists $T_{A}^{ \pm 2} \mathcal{O}_{x}$.

\section{Appendix: Equivalences Respecting $\operatorname{Stab}^{\dagger}(X)$}

Let $\Phi: \mathcal{D}^{b}(X) \rightarrow \mathcal{D}^{b}(Y)$ be a derived equivalence between two K3 surfaces. Recall from section 4 , that $\Phi$ respects the distinguished component if $\Phi_{*} \operatorname{Stab}^{\dagger}(X)=\operatorname{Stab}^{\dagger}(Y)$.

As we will see, this property can be verified for most of the known equivalences. It is expected that $\operatorname{Stab}(X)$ is connected and therefore it should in fact hold always.

We will use the following criterion, which is an easy consequence of Bri08, Prop. 10.3] cf. Proposition 4.6,

Corollary 7.1. Let $\Phi: \mathcal{D}^{b}(X) \rightarrow \mathcal{D}^{b}(Y)$ be a derived equivalence between two K3 surfaces. If the objects $\Phi\left(\mathcal{O}_{x}\right), x \in X$ are $\sigma$-stable of the same phase for some $\sigma \in \operatorname{Stab}^{\dagger}(Y)$, then $\Phi$ preserves the distinguished component.

As a direct application we find the following lemma.

Lemma 7.2. The equivalences of derived categories listed below respect the distinguished component of the stability manifold.

- Shifts: [1] : $A \mapsto A[1]$

- Isomorphisms: For $f: X \cong Y$, the functor $f_{*}: A \mapsto f_{*} A$

- Line bundle twists: For $L \in P i c(X)$, the functor $A \mapsto L \otimes A$

Before we can deal with more interesting auto-equivalences, we need a digression on stability conditions.

7.1. Large volume limit. Following Bri08, Prop. 14.2] we will show that families of Gieseker-stable sheaves give rise to families of $\sigma$-stable objects in stability conditions $\sigma$ near the large volume limit.

Let $M$ be a quasi-compact scheme over $\mathbb{C}$. Denote by $i_{m}: X \rightarrow M \times X$ the inclusion of the fiber over $m \in M(\mathbb{C})$. For a sheaf $E \in \operatorname{Coh}(X \times M)$ denote by $E_{m}$ the restriction $i_{m}^{*} E \in \operatorname{Coh}(X)$ to the fiber over $m$. 
Proposition 7.3. Let $h \in N S(X)$ be an ample class. Let $\mathcal{E} \in \operatorname{Coh}(M \times X)$ be an $M$-flat family of Gieseker-stable sheaves of fixed Mukai vector $v\left(E_{m}\right)=$ $v \in N(X)$. Assume that $r\left(E_{m}\right)>0$ and $\mu\left(E_{m}\right)=\mu_{h}\left(E_{m}\right)>0$.

Then there exists a $n_{0} \geq 1$ such that the objects $E_{m}, m \in M(\mathbb{C})$ are stable with respect to the stability condition $\sigma(0, n h) \in V(X)$ for all $n \geq n_{0}$.

Proof. We will go through Bridgeland's arguments for the case of a single Gieseker-semi-stable sheaf and verify that they suffice to cover our situation. Our presentation fills in some details which were not explicitly mentioned in Bri08.

We first note that the heart $\mathcal{A}(0, n h)$ is independent of $n$ and the objects $E=E_{m}$ lie in the heart $\mathcal{A}(0, n h)$.

Suppose $0 \neq A \rightarrow E$ is a proper sub-object of $E$ in $\mathcal{A}(0, h)$. We have the following formula

$$
\frac{Z_{n}(E)}{r(E)}-\frac{Z_{n}(A)}{r(A)}=-(\nu(E)-\nu(A))+i n(\mu(E)-\mu(A))=: \Delta_{n},
$$

where $\nu(A)=s(A) / r(A)$.

The inequalities $\arg \left(Z_{n}(A)\right)<\arg \left(Z_{n}(E)\right)$ and $\arg \left(Z_{n}(E)\right)<\arg \left(\Delta_{n}\right)$ are equivalent7. Note that we have $Z_{n}(E) \in \mathbb{H}$ by the assumption $\mu(E)>0$, if also $\Delta_{n} \in \mathbb{H}$, then $\arg \left(Z_{n}(E)\right)<\arg \left(\Delta_{n}\right)$ is equivalent to

$$
\frac{\operatorname{Re}\left(Z_{n}(E)\right)}{\operatorname{Im}\left(Z_{n}(E)\right)}>\frac{\operatorname{Re}\left(\Delta_{n}\right)}{\operatorname{Im}\left(\Delta_{n}\right)}=-\frac{\nu(E)-\nu(A)}{n(\mu(E)-\mu(A))} .
$$

We claim that $\Delta_{n} \in \mathbb{H}$ unless $A$ is a subsheaf and $\mu(A)=\mu(E)$, in which case $\Delta_{n} \in \mathbb{R}_{<0}$.

Indeed, consider the exact sequence of cohomology sheaves:

$$
0 \longrightarrow D \longrightarrow A \longrightarrow E \longrightarrow B \longrightarrow 0
$$

where $D=\mathcal{H}^{-1}($ Cone $(A \rightarrow E))$ and $B=\mathcal{H}^{0}(\operatorname{Cone}(A \rightarrow E))$. Note that, $A=\mathcal{H}^{0}(A)$ since $\mathcal{H}^{-1}(A)=\mathcal{H}^{-1}(E)=0$. Let $E^{\prime} \subset E$ be the image of $A \rightarrow E$. We get short exact sequences

$$
0 \longrightarrow D \longrightarrow A \longrightarrow E^{\prime} \longrightarrow 0 \text { and } 0 \longrightarrow E^{\prime} \longrightarrow E \longrightarrow B \longrightarrow 0 \text {. }
$$

If $D=0$, then $A \rightarrow E$ is a subsheaf and $Z_{n}(A) \in \mathbb{H}$ unless $\mu(A)=\mu(E)$. If $D \neq 0$, then we have $\mu(D) \leq 0<\mu(A)$ and therefore also $\mu(A)<\mu\left(E^{\prime}\right)$. Hence $\mu(A)<\mu\left(E^{\prime}\right) \leq \mu(E)$ by stability of $E$. This shows the claim.

In the case $\Delta_{n} \in \mathbb{R}_{<0}$ we always have $\arg \left(Z_{n} E\right)<\arg \left(\Delta_{n}\right)=1$. Thus we may exclude this case henceforth.

Now, the quotient $\operatorname{Re}\left(Z_{n}(E)\right) / \operatorname{Im}\left(Z_{n}(E)\right)$ converges to $+\infty$ for $n \rightarrow \infty$. Hence it suffices to bound $-(\nu(E)-\nu(A)) / n(\mu(E)-\mu(A))$ form above. The numerator can be bounded using the following lemma proved below.

Lemma 7.4. The set

$$
\left\{\nu(A) \mid A \rightarrow E_{m} \text { sub-object in } \mathcal{A}(0, h), m \in M(\mathbb{C})\right\}
$$

is bounded from above.

\footnotetext{
${ }^{7}$ We use the convention that, for $z \in \mathbb{C}^{*}$ the argument $\arg (z)$ is the unique real number in $[0,2)$ such that $z=r \exp (i \pi \arg (z))$ for some $r \in \mathbb{R}_{>0}$.
} 
It remains to find a constant $C$ such that $\mu(E)-\mu(A)>C>0$.

Case $\mu\left(E^{\prime}\right)<\mu(E)$ : As $r\left(E^{\prime}\right) \leq r(E)$ we have $\mu(E)-\mu\left(E^{\prime}\right)>1 / r(E)^{2}$ and since $\mu(A) \leq \mu\left(E^{\prime}\right)$ the same bound holds for $\mu(E)-\mu(A)$ as well.

Case $\mu\left(E^{\prime}\right)=\mu(E)$ : If $D=0$, then $A \rightarrow E$ is a subsheaf and again $\mu(E)-$ $\mu(A)>1 / r(E)^{2}$. If $D \neq 0$, then the exact sequence (8) and $\mu(D) \leq 0$ shows that

$$
\mu(A)=\mu(D) \frac{r(D)}{r\left(E^{\prime}\right)+r(D)}+\mu\left(E^{\prime}\right) \frac{r\left(E^{\prime}\right)}{r\left(E^{\prime}\right)+r(D)} \leq \mu(E) \frac{r(E)}{r(E)+1} .
$$

As $r(E)=r\left(E_{m}\right)$ is independent of $m$ we get a uniform bound.

Proof of Lemma. Recall that if $v(A)=(r, l, s)$, then $\nu(A)=s / r$. The Eulercharacteristic of $A$ is computed as

$$
h^{0}(A)-h^{1}(A)+h^{2}(A)=\chi(A)=\chi\left(\mathcal{O}_{X}, A\right)=-(1,0,1) .(r, l, s)=r+s
$$

hence it suffices to bound $\chi(A) / r$ from above. As $\mu^{\min }(A)>0=\mu\left(\mathcal{O}_{X}\right)$ we have $\operatorname{Hom}\left(A, \mathcal{O}_{X}\right)=H^{2}(A)=0$. Therefore $\chi(A) / r \leq h^{0}(A) / r$. The long exact sequence

$$
0 \longrightarrow H^{0}(D) \longrightarrow H^{0}(A) \longrightarrow H^{0}\left(E^{\prime}\right) \longrightarrow H^{1}(D) \longrightarrow \ldots
$$

shows that $h^{0}(A) \leq h^{0}(D)+h^{0}\left(E^{\prime}\right)$. Moreover, $h^{0}\left(E^{\prime}\right) \leq h^{0}(E)$ and $h^{0}(E)=$ $h^{0}\left(E_{m}\right)$ is bounded uniformly in $m \in M(\mathbb{C})$, since $h^{0}\left(E_{m}\right)$ is semi-continuous and $M$ quasi-compact. In the case $D=0$ we are done. Let now $D \neq 0$. Note that $r=r(A) \geq r(D)$, and hence $h^{0}(D) / r(A) \leq h^{0}(D) / r(D)$. Therefore it suffices to bound

$$
\left\{h^{0}(D) / r(D) \mid D=\mathcal{H}^{-1}\left(\text { Cone }\left(A \rightarrow E_{m}\right)\right), m \in M(\mathbb{C})\right\} .
$$

Claim: $h^{0}(D) / r(D) \leq 1$ for all $D \neq 0$ torsion free with $\mu^{\text {min }}(D) \leq 0$.

Let $0 \neq s \in H^{0}(D)$ be a section. As in [Bri08, Lem. 14.3] we show that $s: \mathcal{O}_{X} \rightarrow D$ is injective and that $Q=\operatorname{coker}(s)$ is torsion free. Note that also $\mu^{\min }(Q) \geq \mu^{\min }(D) \geq 0$ unless $Q=0$. Hence $Q$ fulfills the same assumptions as $D$ and we can apply induction on $r(D)$.

If $r(D)=1$, then $h^{0}(D)=0$ as $D$ has no non-trivial torsion-free quotients. If $r(D)>1$, then $h^{0}(D)=h^{0}(Q)+1$, since $h^{1}\left(X, \mathcal{O}_{X}\right)=0$, and therefore

$$
\frac{h^{0}(D)}{r(D)}=\frac{h^{0}(Q)+1}{r(Q)+1} \leq 1
$$

by induction hypothesis.

7.2. Moduli spaces and spherical twists. A very important class of derived equivalences between K3 surfaces is provided by moduli spaces of sheaves.

Proposition 7.5. Let $M=M_{h}(v)$ be a fine, compact, two-dimensional moduli-space of Gieseker-stable sheaves on $X$ and $\Phi: \mathcal{D}^{b}(M) \stackrel{\sim}{\longrightarrow} \mathcal{D}^{b}(X)$ the Fourier-Mukai equivalence induced by the universal family (cf. Huy06, Sec. 10.2]).

Then $\Phi$ respects the distinguished component. 
Proof. Let $\mathcal{O}(1)$ be the ample line bundle with $c_{1}(\mathcal{O}(1))=h$. Tensoring with a large power of $\mathcal{O}(1)$ we reduce to the case $\mu(E)>0$. Now Proposition 7.3 applies and there is an $n>0$ such that all the sheaves $E_{m}, m \in M(\mathbb{C})$ are stable in the stability condition $\sigma(0, n h) \in U(X)$. As all sheaves $E_{m}$ lie in the heart $\mathcal{A}(0, h)$ they have the same phase. Hence the proposition follows from Corollary 7.1 .

Proposition 7.6. Let $A$ be a spherical vector bundle, which is Giesekerstable with respect to an ample class $h \in N S(X)$.

Then the spherical twist $T_{A}: \mathcal{D}^{b}(X) \rightarrow \mathcal{D}^{b}(X)$ respects the distinguished component.

Proof. The spherical twist functor has Fourier-Mukai kernel

$$
\mathcal{P}=\text { Cone }\left(p r_{1}^{*} A^{\vee} \otimes p r_{2}^{*} A \stackrel{t r}{\longrightarrow} \mathcal{O}_{\Delta}\right) \in \mathcal{D}^{b}(X \times X)
$$

cf. Huy06, Def. 8.3]. Let $i_{x}: X \rightarrow\{x\} \times X \subset X \times X$ be the inclusion of the fiber. Since $i_{x}^{*}(\mathcal{P})[1]=T_{A}\left(\mathcal{O}_{x}\right)[1]=: E_{x}$ is a sheaf, the complex $\mathcal{P}[1]$ is quasi isomorphic to a sheaf which is flat along $\mathrm{pr}_{1}$.

Mukai shows in [Muk87, Rem. 3.11.] that the sheaves $E_{x}$ are Giesekerstable. Therefore $\mathcal{P}[1]$ induces a map $f: X \rightarrow M_{h}\left(v_{1}\right)$, where $v_{1}=$ $v\left(T_{A}\left(\mathcal{O}_{x}\right)\right)$, which is easily seen to be an isomorphism. Hence $T_{A}$ is a special case of a Fourier-Mukai transformation at a moduli space of Gieseker-stable sheaves and Proposition [7.5 applies.

Using Bridgeland's description the boundary of $U(X)$ in Bri08, Thm. 12.1] (cf. Theorem 4.9) we can also show that spherical twists along torsion sheaves do respect the distinguished component.

Proposition 7.7. Let $C$ be a (-2)-curve on a K3 surface $X$ and $k \in \mathbb{Z}$, then the spherical twist $T_{\mathcal{O}_{C}(k)}$ does respect the distinguished component.

Proof. We will show that every pair $(C, k)$ does define a non-empty boundary component of $U(X)$ of type $\left(C_{k}\right)$. Then [Bri08, Thm. 12.1] shows that $T_{\mathcal{O}_{C}(k)_{*}} \bar{U}(X) \cap \bar{U}(X) \neq \emptyset$ and therefore $T_{\mathcal{O}_{C}(k)}$ respects the distinguished component, cf. Remark 4.13 .

Every $(-2)$-curve $C$ defines a boundary component of the ample cone, i.e. there is a class $\eta \in \overline{A m p(X)}$ such that $C . \eta=0$ and $C^{\prime} . \eta>0$ for all other $(-2)$-curves $C^{\prime}$. Multiplying with a positive number we can assume that $\eta^{2}>2$.

We claim that there is always a $\beta \in N S(X)_{\mathbb{R}}$ such that

(1) $\exp (i \eta+\beta) . \delta \neq 0$ for all $\delta \in \Delta(X)$, i.e. $\exp (i \eta+\beta) \in \mathcal{P}_{0}^{+}(X)$.

(2) $\exp (i \eta+\beta) . \delta \notin \mathbb{R}_{\leq 0}$ for all $\delta \in \Delta^{>0}(X)$ and

(3) $\beta . C+k \in(-1,0)$.

Indeed, for $\delta=(r, l, s)$ we have

$$
\operatorname{Im}(\exp (i \eta+\beta) . \delta)=l . \eta-r \beta . \eta .
$$

This number is non-zero if $r \neq 0$ and $\beta . \eta \neq l . \eta / r$. If $r=0$, then $\delta^{2}=l^{2}=-2$ and $\exp (i \eta+\beta) . \delta=l . \eta=0$ implies that $l= \pm C$ by construction of $\eta$. In this case $\operatorname{Re}(\exp (i \eta+\beta) \cdot \delta)= \pm \beta . C-s$ is nonzero if (3) is fulfilled. Thus it suffices to chose $\beta$ in such a way that the countably many inequalities 
$\beta . \eta \neq l . \eta / r, l \in N S(X)$ and the open condition (3) hold. This shows the claim.

Let $\sigma$ be the unique stability condition in $\bar{U}(X)$ with central charge $\exp (i \eta+\beta)$. Note that $\sigma$ does not lie on a boundary component of type $\left(A^{ \pm}\right)$by (2). By construction, if $x \in C$ then $\mathcal{O}_{x}$ is destabilized by a sequence

$$
0 \longrightarrow \mathcal{O}_{C}(n+1) \longrightarrow \mathcal{O}_{x} \longrightarrow \mathcal{O}_{C}(n)[1] \longrightarrow 0
$$

Thus $\sigma$ is a general point of a boundary component of $U(X)$ of type $\left(C_{n}\right)$ for some $n$. The number $n$ is uniquely determined by the property that

$$
Z_{\sigma}\left(\mathcal{O}_{C}(n)[1]\right), Z_{\sigma}\left(\mathcal{O}_{C}(n+1)\right) \in \mathbb{H} \cup \mathbb{R}_{<0}
$$

which has to hold since $\mathcal{O}_{C}(n+1)$ and $\mathcal{O}_{C}(n)[1]$ lie in the heart of $\sigma$. This is equivalent to $-1 \leq \beta . C+n<0$. Hence $k=n$ by condition (3).

Remark 7.8. The general question if for a spherical object $A \in \mathcal{D}^{b}(X)$ the equivalence $T_{A}$ respects the distinguished component remains open - even in the case that $A$ is a vector bundle.

7.3. Auto-equivalences and the Kähler moduli space. It was shown by [HLOY04, Plo05] and HMS09. (cf. Theorem 3.1) that the image of the map

$$
\operatorname{Aut}\left(\mathcal{D}^{b}(X)\right) \longrightarrow O_{\text {Hodge }}(\tilde{H}(X, \mathbb{Z}))
$$

is the index two subgroup $O_{\text {Hodge }}^{+}(\tilde{H}(X, \mathbb{Z}))$.

Proposition 7.9. Let $A u t^{\dagger}\left(\mathcal{D}^{b}(X)\right) \subset \operatorname{Aut}\left(\mathcal{D}^{b}(X)\right)$ be the subgroup of autoequivalences which respect the distinguished component. Then

$$
A u t^{\dagger}\left(\mathcal{D}^{b}(X)\right) \longrightarrow O_{\text {Hodge }}^{+}(\tilde{H}(X, \mathbb{Z}))
$$

is surjective.

Proof. As explained in Huy06, Cor. 10.13.] every element of $O_{\text {Hodge }}^{+}(\tilde{H}(X, \mathbb{Z}))$ is induced by the composition of derived equivalences of the following type.

(1) Line bundle twists: For $L \in \operatorname{Pic}(X)$, the functor $L \otimes_{-} \in \operatorname{Aut}\left(\mathcal{D}^{b}(X)\right)$.

(2) For isomorphisms $f: X \rightarrow Y$, the functor $f_{*}: \mathcal{D}^{b}(X) \rightarrow \mathcal{D}^{b}(Y)$.

(3) For fine, compact, two-dimensional moduli spaces $M$ of Giesekerstable sheaves with universal family $\mathcal{E}$, the Fourier-Mukai transform

$$
F M(\mathcal{E}): \mathcal{D}^{b}(M) \rightarrow \mathcal{D}^{b}(X) .
$$

(4) Spherical twists along $\mathcal{O}_{X}$.

(5) Spherical twists along $\mathcal{O}_{C}$ for a $(-2)$-curve $C \subset X$.

All these equivalences do respect the distinguished component due to our Lemma 7.2 for (1),(2), Proposition 7.5 for (3), Proposition 7.6 for (4) and Proposition 7.7 for (5).

This result enables us prove the alternative description of the Kähler moduli space using the stability manifold, alluded to in Remark 3.4. We use the notation from Section 2 and 3.

Corollary 7.10. We have

$$
A u t^{\dagger}\left(\mathcal{D}^{b}(X)\right) \backslash S t a b^{\dagger}(X) / \widetilde{G l} l_{2}^{+}(\mathbb{R}) \cong K M_{0}(X)
$$

where $K M_{0}(X)=\Gamma_{X} \backslash \mathfrak{D}_{0}(N(X)) \subset K M(X)$. 
Proof. Recall that $A u t_{0}^{\dagger}\left(\mathcal{D}^{b}(X)\right)$ is the subgroup of $A u t^{\dagger}\left(\mathcal{D}^{b}(X)\right)$ of autoequivalences acting trivially on $\tilde{H}(X, \mathbb{Z})$. By [Bri08, Thm. 1.1] (cf. Theorem 4.1) the quotient $A u t_{0}^{\dagger}\left(\mathcal{D}^{b}(X)\right) \backslash \operatorname{Stab}^{\dagger}(X)$ is identified with the period domain $\mathcal{P}_{0}^{+}(X) \subset N(X)_{\mathbb{C}}$ via $\pi: \operatorname{Stab}^{\dagger}(X) \rightarrow \mathcal{P}_{0}^{+}(X)$. As $\pi$ is $\widetilde{G l} l_{2}^{+}(\mathbb{R})$ equivariant, we have

$$
A u t_{0}^{\dagger}\left(\mathcal{D}^{b}(X)\right) \backslash \operatorname{Stab}^{\dagger}(X) / \widetilde{G l}{ }_{2}^{+}(\mathbb{R}) \cong \mathcal{P}_{0}^{+}(X) / G l_{2}^{+}(\mathbb{R}) \cong \mathfrak{D}_{0}^{+}(X) .
$$

Now Proposition 7.9 shows that

$$
A u t^{\dagger}\left(\mathcal{D}^{b}(X)\right) \backslash \mathfrak{D}_{0}^{+}(X) \cong O_{\text {Hodge }}^{+}(\tilde{H}(X, \mathbb{Z})) \backslash \mathfrak{D}_{0}^{+}(X) \cong K M_{0}(X) .
$$

7.4. Reduction to the large volume limit. As another consequence we obtain the following proposition which allows us to reduce many statements about objects with a standard Mukai vector $v$ (cf. Definition 3.7) to the special case $v=v_{0}=(0,0,1) \in N(X)$, which is the class of a point sheaf.

Proposition 7.11. Let $v \in N(X)$ be a standard vector. Then there is a K3 surface $Y$ and a derived equivalence $\Phi: \mathcal{D}^{b}(X) \rightarrow \mathcal{D}^{b}(Y)$ such that

$$
\Phi^{H}(v)=v_{0}
$$

and $\Phi$ respects the distinguished component.

Moreover, $Y$ is a fine moduli space of Gieseker-stable sheaves and the Hodge structure $H^{2}(Y, \mathbb{Z})$ isomorphic to the subquotient $v^{\perp} / v$ of $\tilde{H}(X, \mathbb{Z})$.

Proof. Write $v=(r, l, s)$. Applying $T_{\mathcal{O}_{X}}$, shifts and $L \otimes_{-}$for a line bundle $L$ as in the proof of [Huy06, 10.10] we reduce to the case that that $r>0$. Note that the equivalences $T_{\mathcal{O}_{X}},[1], L \otimes$ - respect the distinguished component by Lemma 7.2 and Proposition 7.6 ,

By [Huy06, Sec. 10.2] there is an ample class $h \in N S(X)$ such that the moduli space of Gieseker-stable sheaves $Y=M_{h}(v)$ is a K3 surface with Hodge structure $H^{2}(Y, \mathbb{Z}) \cong v^{\perp} / v$ as subquotient of $\tilde{H}(X, \mathbb{Z})$. The derived equivalence induced by the universal bundle $\mathcal{E}$ respects the distinguished component by Proposition 7.5 and maps $v_{0}$ to $v$.

\section{REFERENCES}

[BB66] Walter L. Baily and Armand Borel. Compactification of arithmetic quotients of bounded symmetric domains. Ann. of Math. (2), 84:442-528, 1966.

[BBD85] Arnaoud Beauville, Jean-Pierre Bourguignon, and Michel Demazure. Géométrie des surfaces K3: modules et périodes. Société Mathématique de France, Paris, 1985.

[BJ06] Armand Borel and Lizhen Ji. Compactifications of symmetric and locally symmetric spaces. Mathematics: Theory \& Applications. Birkhäuser Boston Inc., Boston, MA, 2006.

[Bri07] Tom Bridgeland. Stability conditions on triangulated categories. Ann. of Math. (2), 166(2):317-345, 2007.

[Bri08] Tom Bridgeland. Stability conditions on $K 3$ surfaces. Duke Math. J., 141(2):241-291, 2008.

[Bri09] Tom Bridgeland. Spaces of stability conditions. In Algebraic geometry-Seattle 2005. Part 1, volume 80 of Proc. Sympos. Pure Math., pages 1-21. Amer. Math. Soc., Providence, RI, 2009.

[Dol96] Igor V. Dolgachev. Mirror symmetry for lattice polarized K3 surfaces. J. Math. Sci., 81(3):2599-2630, 1996. 
[Gro71] Alexander Grothendieck. Théorie des intersections et théorème de RiemannRoch (SGA6). Lecture Notes in Mathematics, Vol. 225. Springer-Verlag, Berlin, 1971.

[Har77] Robin Hartshorne. Algebraic geometry. Springer-Verlag, New York, 1977. Graduate Texts in Mathematics, No. 52.

[Hel78] Sigurdur Helgason. Differential geometry, Lie groups, and symmetric spaces, volume 80 of Pure and Applied Mathematics. Academic Press Inc. [Harcourt Brace Jovanovich Publishers], New York, 1978.

[HLOY04] Shinobu Hosono, Bong H. Lian, Keiji Oguiso, and Shing-Tung Yau. Auto equivalences of derived category of a $K 3$ surface and monodromy transformations. J. Algebraic Geom., 13(3):513-545, 2004.

[HMS09] Daniel Huybrechts, Emanuele Macrì, and Paolo Stellari. Derived equivalences of K3 surfaces and orientation. Duke Math. J., 149(3):461-507, 2009.

[Huy06] Daniel Huybrechts. Fourier-Mukai transforms in algebraic geometry. Oxford Mathematical Monographs. The Clarendon Press Oxford University Press, Oxford, 2006.

[Huy08] Daniel Huybrechts. Derived and abelian equivalence of $K 3$ surfaces. J. Algebraic Geom., 17(2):375-400, 2008.

[JM02] L. Ji and R. MacPherson. Geometry of compactifications of locally symmetric spaces. Ann. Inst. Fourier (Grenoble), 52(2):457-559, 2002.

[Lie06] Max Lieblich. Moduli of complexes on a proper morphism. J. Algebraic Geom., 15(1):175-206, 2006.

[Loo03] Eduard Looijenga. Compactifications defined by arrangements. II. Locally symmetric varieties of type IV. Duke Math. J., 119(3):527-588, 2003.

[Ma09] Shouhei Ma. Fourier-Mukai partners of a $K 3$ surface and the cusps of its Kahler moduli. Internat. J. Math., 20(6):727-750, 2009.

[Ma10] Shouhei Ma. On the 0-dimensional cusps of the Kähler moduli of a $K 3$ surface. Math. Ann., 348(1):57-80, 2010.

[Muk87] Shigeru Mukai. On the moduli space of bundles on K3 surfaces. I, volume 11 of Tata Inst. Fund. Res. Stud. Math. Tata Inst. Fund. Res., Bombay, 1987.

[Plo05] David Ploog. Groups of autoequivalences of derived categories of smooth projective varieties. $\mathrm{PhD}$ thesis, Berlin, 2005.

[Tod08] Yukinobu Toda. Moduli stacks and invariants of semistable objects on $K 3$ surfaces. Adv. Math., 217(6):2736-2781, 2008. 